\title{
Singular extensions and the second cohomology categorical group
}

\author{
A. R. Garzón ${ }^{1}$
}

Received: 21 September 2015 / Accepted: 3 May 2016 / Published online: 23 May 2016

(C) Tbilisi Centre for Mathematical Sciences 2016

\begin{abstract}
We give a classification of the 2-groupoid of singular extensions of categorical groups by means of a second cohomology categorical group, whose definition uses the notion of nerve of a categorical group. This second cohomology is, then, shown to be involved in a five term 2-exact sequence à la Hochschild-Serre which is associated with any essentially surjective homomorphism of categorical groups.
\end{abstract}

Keywords (Symmetric) categorical group - Categorical action · Non-functorial derivation $\cdot 2$-exactness $\cdot$ Cohomology categorical group $\cdot$ Singular extension

Mathematics Subject Classification 18D10 - 18B40 - 18D35 - 18G50 - 20J06

\section{Introduction}

For any group $G$ and any $G$-module $A$, it is well-known that the abelian group of singular extensions of $G$ by $A$ is classified by the second Eilenberg-Mac Lane cohomology group $H^{2}(G, A)$ [19]. Such a cohomological classification, for singular extensions of categorical groups with symmetric kernel and a functorial section, has been recently showed in [13]. This classification uses a second categorical cohomology group whose definition generalizes the cohomology groups defined by Ulbrich in [23]. It is obtained

Communicated by Nick Inassaridze.

This work has been partially supported by MCI of Spain (Project: MTM2011-22554); Consejería de Innovación de J. de Andalucía (FQM-168).

$凶$ A. R. Garzón

agarzon@ugr.es

1 Departamento de Álgebra, Facultad de Ciencias, Universidad de Granada, 18071 Granada, Spain 
from a cochain complex of symmetric categorical groups that is built through the pseudo-simplicial category that $\mathbb{G}$ defines by the familiar bar construction (see $[4,8]$ ), but taking into account the $\mathbb{G}$-action on $\mathbb{A}$.

The aim in this paper is to give the same answer to the more general problem of considering all singular extensions of categorical groups with symmetric kernel. In other words, to give a cohomological classification of the symmetric categorical group of singular extensions of a categorical group $\mathbb{G}$ by a given symmetric $\mathbb{G}$-categorical group $\mathbb{A}$. This is carried out in Sect. 3 and, for this classification, we introduce a second cohomology categorical group $\mathcal{H}^{2}(\mathbb{G}, \mathbb{A})$ which is obtained from a cochain complex of symmetric categorical groups that is built through the simplicial set, $\operatorname{Ner}(\mathbb{G})$, nerve of a categorical group $\mathbb{G}$ introduced in [5]. We, then, prove in Theorem 3.5 the existence of a biequivalence of bigroupoids giving the desired classification and, moreover, determining in Corollary 3.6 a Baer sum in the groupoid of singular extensions.

With the objective of suggesting a proof for a categorical-group version of the classical Hochschild-Serre 5-term exact sequence, we developed in Section 2 of [13] a new proof, suitable to our 2-dimensional interest, of this group exact sequence. With this precedent, and using the second categorical cohomology group there defined, we showed, in Section 6 of [13], Hoschild-Serre's sequences for categorical groups. Now, in Sect. 4, as a main application of this paper, and in a parallel way to that developed in [13], we show new categorical group versions of the classical Hochschild-Serre 5-term exact group sequences [14] involving the $\mathcal{H}^{2}$ here studied. These sequences are associated to any essentially surjective homomorphism of categorical groups and, in them, new categorical groups of 'non-functorial derivations', which are introduced at the beginning of the section, appear.

Firstly, before these two sections, we collect in Sect. 2 some basic facts on (symmetric) categorical groups (see $[1,3,16,21]$ for the background) and on the corresponding notions of action [9, 10,12], kernel, cokernel, and exactness [10,18,24]. Later, we also recall some results about categorical crossed modules (see [10]).

\section{Preliminaries}

A categorical group (sometimes called gr-category or 2-group in the literature) is a monoidal groupoid $\mathbb{G}=(\mathbb{G}, \otimes, I, a, l, r)$ such that every object $X$ is invertible, that is, the functor

$$
X \otimes(-): \mathbb{G} \rightarrow \mathbb{G}, \quad Y \mapsto X \otimes Y
$$

is an equivalence. It is then possible to choose, for each $X \in \mathbb{G}$, an object $X^{*} \in \mathbb{G}$ (called an inverse of $X$ and denoted in this way following [16] or [3]) and arrows $\eta_{X}: I \rightarrow X \otimes X^{*}$ and $\varepsilon_{X}: X^{*} \otimes X \rightarrow I$ such that the usual triangular identities are satisfied. The choice of a system of inverses $\left(X^{*}, \eta_{X}, \epsilon_{X}\right), X \in \mathbb{G}$, induces a categorical equivalence

$$
(-)^{*}: \mathbb{G} \rightarrow \mathbb{G} \quad f: X \rightarrow Y \mapsto f^{*}: X^{*} \rightarrow Y^{*}
$$

where $f^{*}$ is defined as follows 


$$
X^{*} \simeq X^{*} \otimes I \stackrel{1 \otimes \eta_{Y}}{\longrightarrow} X^{*} \otimes Y \otimes Y^{*} \stackrel{1 \otimes f^{-1} \otimes 1}{\longrightarrow} X^{*} \otimes X \otimes Y^{*} \stackrel{\varepsilon_{X} \otimes 1}{\longrightarrow} I \otimes Y^{*} \simeq Y^{*}
$$

Note that, if in the definition of $f^{*}$ we use $f$ instead of $f^{-1}$, then the equivalence $(-)^{*}: \mathbb{G} \longrightarrow \mathbb{G}$ is contravariant and $\left(X, X^{*}, \eta_{X}, \varepsilon_{X}\right)$ is a duality in $\mathbb{G}$.

A categorical group $\mathbb{G}$ is said to be symmetric if it is symmetric as a monoidal category, the symmetry being usually denoted by $c_{X, Y}: X \otimes Y \rightarrow Y \otimes X$. We will denote by $\mathcal{C G}$ (respectively $\mathcal{S C G}$ ) the 2-category whose objects are categorical groups (respectively, symmetric categorical groups). Following the terminology in [16], the 1arrows, which are monoidal functors $T=\left(T, T_{2}\right): \mathbb{G} \rightarrow \mathbb{H}$ (respectively, symmetric monoidal functors), are called homomorphisms, and the 2-arrows, which are monoidal natural transformations, are called morphisms. Note that a canonical arrow $T_{0}: I \rightarrow$ $T I$ can be constructed from the natural and coherent family of arrows $T_{2}^{X, Y}: T X \otimes$ $T Y \rightarrow T(X \otimes Y)$. Note also that in $\mathcal{C G}$ and $\mathcal{S C G}$, the 2-arrows are invertible.

When no confusion arises, in order to simplify notation we will

- omit the associativity isomorphism $a_{X, Y, Z}:(X \otimes Y) \otimes Z \rightarrow X \otimes(Y \otimes Z)$ and the right and left unit isomorphisms $r_{X}: X \rightarrow X \otimes I$ and $l_{X}: X \rightarrow I \otimes X$

- omit indexes, writing for example $c: X \otimes Y \rightarrow Y \otimes X$ instead of $c_{X, Y}$

- denote canonical arrows as "can" or even as unlabelled arrows; for example, we write can : $X \rightarrow Y \otimes X \otimes Y^{*}$ or just $X \simeq Y \otimes X \otimes Y^{*}$ instead of

$$
X \stackrel{r_{X}}{\longrightarrow} X \otimes I \stackrel{1 \otimes \eta_{Y}}{\longrightarrow} X \otimes Y \otimes Y^{*} \stackrel{c_{X, Y} \otimes 1}{\longrightarrow} Y \otimes X \otimes Y^{*} .
$$

If $\mathbb{G}$ and $\mathbb{A}$ are categorical groups, an action of $\mathbb{G}$ on $\mathbb{A}$ is a homomorphism of categorical groups $\varphi: \mathbb{G} \rightarrow \mathbb{E q}(\mathbb{A})$, where $\mathbb{E q}(\mathbb{A})$ is the categorical group of monoidal autoequivalences of $\mathbb{A}$. When such a $\mathbb{G}$-action is given, we will say that $\mathbb{A}=(\mathbb{A}, \varphi)$ is a $\mathbb{G}$-categorical group. To give a $\mathbb{G}$-categorical group structure on $\mathbb{A}$ is equivalent to giving a functor

$$
\varphi: \mathbb{G} \times \mathbb{A} \rightarrow \mathbb{A}, \quad(X, A) \stackrel{(f, u)}{\longrightarrow}(Y, B) \mapsto \varphi(X)(A)={ }^{X} A \stackrel{\varphi(f)(u)={ }^{f} u}{\longrightarrow} \varphi(Y)(B)={ }^{Y} B
$$

(when $f=\operatorname{id}_{X}$ or $u=\operatorname{id}_{A}$, we write respectively $X_{u \text { and }}{ }^{f} A$ instead of $f_{u}$ ) together with natural families of arrows

$$
\varphi_{2}^{X, A, B}:{ }^{X} A \otimes{ }^{X} B \rightarrow{ }^{X}(A \otimes B), \quad \varphi_{1}^{X, Y, A}:{ }^{X}\left({ }^{Y} A\right) \rightarrow{ }^{X \otimes Y} A, \quad \varphi_{0}^{A}: A \rightarrow{ }^{I} A
$$

satisfying suitable coherence conditions (see Remark 3.5 in [13] or Definition 2.1 in [9]). ${ }^{1}$

We say that an action $\varphi: \mathbb{G} \rightarrow \mathbb{E q}(\mathbb{A})$ is symmetric (or that $\mathbb{A}$ is a symmetric $\mathbb{G}$-categorical group) if $\mathbb{A}$ is symmetric and $\varphi$ factorizes through $\varphi: \mathbb{G} \rightarrow \mathbb{E} \mathrm{q}_{s}(\mathbb{A})$, where $\mathbb{E} \mathrm{q}_{s}(\mathbb{A})$ is the categorical group of symmetric monoidal autoequivalences of $\mathbb{A}$.

\footnotetext{
1 All along the paper, we will consider several coherence conditions. When they are already available in the literature, we limit ourselves to give a precise reference. Otherwise, we express the condition as an equation or in the more readable form of a commutative diagram.
} 
Actions form a 2-category, denoted $\mathcal{A C T}$ (or $\mathcal{S} \mathcal{A C T}$ if we restrict to symmetric actions), where the 1-cells are the homomorphisms of actions, that is, triples

$$
(R, F, \lambda):(\mathbb{G}, \mathbb{A}, \varphi) \rightarrow\left(\mathbb{G}^{\prime}, \mathbb{A}^{\prime}, \varphi^{\prime}\right)
$$

with $R: \mathbb{G} \rightarrow \mathbb{G}^{\prime}$ and $F: \mathbb{A} \rightarrow \mathbb{A}^{\prime}$ two homomorphisms in $\mathcal{C G}$ (with $F$ in $\mathcal{S C G}$ if the actions $\varphi$ and $\varphi^{\prime}$ are symmetric), and

$$
\lambda^{X, A}: F\left({ }^{X} A\right) \rightarrow{ }^{R X} F A
$$

a natural family of arrows satisfying suitable coherence conditions (see [13]).

Given two homomorphisms of actions

$$
(R, F, \lambda),\left(R^{\prime}, F^{\prime}, \lambda^{\prime}\right):(\mathbb{G}, \mathbb{A}, \varphi) \rightarrow\left(\mathbb{G}^{\prime}, \mathbb{A}^{\prime}, \varphi^{\prime}\right)
$$

a morphism (2-cell) of actions is a pair of morphisms

$$
\beta: R \Rightarrow R^{\prime}, \quad \alpha: F \Rightarrow F^{\prime}
$$

in $\mathcal{C G}$ compatibles with the actions (see [13]).

Given a homomorphism $T: \mathbb{G} \rightarrow \mathbb{H}$ in $\mathcal{C G}$, its kernel is the following diagram in $\mathcal{C G}$

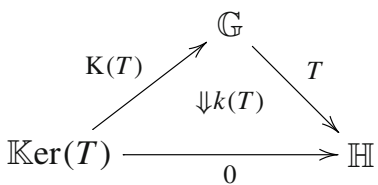

where

- an object of $\mathbb{K e r}(T)$ is a pair $(X \in \mathbb{G}, x: T X \rightarrow I)$;

- an arrow $f:(X, x) \rightarrow(Y, y)$ in $\mathbb{K e r}(T)$ is an arrow $f: X \rightarrow Y$ in $\mathbb{G}$ such that $y \cdot T(f)=x$;

- the faithful (but in general not full) homomorphism $\mathrm{K}(T): \mathbb{K} \operatorname{er}(T) \rightarrow \mathbb{G}$ is defined by $\mathrm{K}(T)(f:(X, x) \rightarrow(Y, y))=(f: X \rightarrow Y)$;

- the component at $(X, x)$ of the morphism $k(T)$ is given by $x: T(\mathrm{~K}(T)(X, x))=$ $T X \rightarrow I=0(X, x)$.

The kernel of $T$ is a bilimit in the sense of [2] and also a standard homotopy kernel (see [18]).

Note that, because of the double universal property of the kernel, we do not pay too much attention to the fact that a diagram in $\mathcal{C G}$ or in $\mathcal{S C G}$ involving kernels (or cokernels) commutes strictly or just up to a 2-arrow. 
Let now $T: \mathbb{G} \rightarrow \mathbb{H}$ be a homomorphism in $\mathcal{S C G}$. Its cokernel is the following diagram in $\mathcal{S C G}$

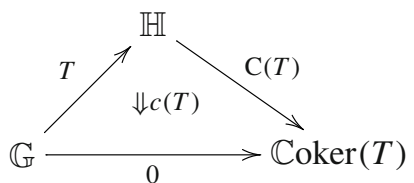

and it satisfies two universal properties dual to those of the kernel. It can be described as follows:

- the objects of $\operatorname{Coker}(T)$ are those of $\mathbb{H}$;

- a prearrow from $A$ to $B$ is a pair $(X \in \mathbb{G}, f: A \rightarrow T X \otimes B)$;

- an arrow $[X, f]: A \longrightarrow B$ is an equivalence class of prearrows, where two prearrows $(X, f),\left(X^{\prime}, f^{\prime}\right)$ from $A$ to $B$ are equivalent if there exists an arrow $x: X \rightarrow X^{\prime}$ in $\mathbb{G}$ such that $(T(x) \otimes 1) \cdot f=f^{\prime}$;

- the tensor product of two arrows $[X, f]: A \longrightarrow B$ and $[Y, g]: C \longrightarrow D$ is given by the class of the prearrow with object part $X \otimes Y$ and arrow part

$A \otimes C \stackrel{f \otimes g}{\longrightarrow} T X \otimes B \otimes T Y \otimes D \stackrel{1 \otimes c \otimes 1}{\longrightarrow} T X \otimes T Y \otimes B \otimes D \simeq T(X \otimes Y) \otimes B \otimes D$

- the essentially surjective on objects homomorphism $\mathrm{C}(T): \mathbb{H} \rightarrow \operatorname{Coker}(T)$ sends an arrow $f: A \rightarrow B$ to the class of the prearrow

$$
(I, A \stackrel{f}{\longrightarrow} B \longrightarrow T I \otimes B)
$$

$\mathrm{C}(T)$ in general is not a full functor;

- the component at $X \in \mathbb{G}$ of the morphism $c(T)$ is given by the class of the prearrow $(X, T X \rightarrow I \otimes T X)$.

Note that, although we have described the cokernel $\operatorname{Coker}(T)$ as a categorical group, in fact it is obtained from a monoidal bicategory by taking 2-isomorphism classes of 1 -arrows as arrows. This fact that $\operatorname{Coker}(T)$ is a monoidal bicategory is mentioned in the introduction of [24], and the whole proof has been done in [15].

A diagram

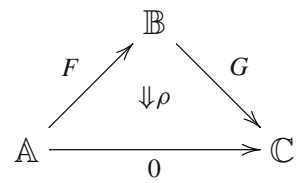

in $\mathcal{C G}$ is 2-exact if the comparison homomorphism $F^{\prime}: \mathbb{A} \rightarrow \mathbb{K e r}(G)$ is full and essentially surjective on objects. When the above diagram is in $\mathcal{S C G}$, its 2-exactness is equivalent to asking that the comparison homomorphism $G^{\prime}: \operatorname{Coker}(F) \rightarrow \mathbb{C}$ is 
full and faithful. Obvious examples of 2-exact sequences are provided by the above kernel and cokernel constructions.

Categorical precrossed modules and categorical crossed modules have been introduced in [10], and used in [7] as algebraic models for connected homotopy 3-types. Next we recall the construction of the 2-category of categorical crossed modules and some results on the quotient categorical group associated with a categorical crossed module that we will use below (c.f. $[10,13]$ )

A categorical crossed module (see Definition 2.2 and 2.4 in [10]) is a 6-tuple

$$
(\mathbb{G}, \mathbb{A}, \varphi: \mathbb{G} \rightarrow \mathbb{E q}(\mathbb{A}), T: \mathbb{A} \rightarrow \mathbb{G}, \nu, \chi)
$$

with $(\mathbb{G}, \mathbb{A}, \varphi)$ an action, $T: \mathbb{A} \rightarrow \mathbb{G}$ a homomorphism in $\mathcal{C G}$, and

$$
v_{X, A}: T\left({ }^{X} A\right) \otimes X \rightarrow X \otimes T A \text { and } \chi_{A, B}:{ }^{T A} B \otimes A \rightarrow A \otimes B
$$

natural families of arrows satisfying suitable coherence conditions.

A homomorphism from $(\mathbb{G}, \mathbb{A}, \varphi, T, v, \chi)$ to $\left(\mathbb{G}^{\prime}, \mathbb{A}^{\prime}, \varphi^{\prime}, T^{\prime}, v^{\prime}, \chi^{\prime}\right)$ is a 4-tuple $(R, F, \lambda, \tau)$ with $(R, F, \lambda):(\mathbb{G}, \mathbb{A}, \varphi) \rightarrow\left(\mathbb{G}^{\prime}, \mathbb{A}^{\prime}, \varphi^{\prime}\right)$ a homomorphism in $\mathcal{A C T}$, and $\tau: R \cdot T \Rightarrow T^{\prime} \cdot F$ a morphism in $\mathcal{C G}$ satisfying suitable coherence conditions. Finally, if $(R, F, \lambda, \tau)$ and $\left(R^{\prime}, F^{\prime}, \lambda^{\prime}, \tau^{\prime}\right)$ are two such homomorphisms of categorical crossed modules, a morphism of categorical crossed modules is a morphism

$$
(\beta, \alpha):(R, F, \lambda) \Rightarrow\left(R^{\prime}, F^{\prime}, \lambda^{\prime}\right)
$$

in $\mathcal{A C T}$ satisfying again a suitable coherence condition.

Categorical crossed modules with their homomorphisms and morphisms form a 2-category denoted $\mathcal{C C M}$ (or $\mathcal{S C C M}$ if we restrict to symmetric actions). Whenever the homomorphism $T: \mathbb{A} \rightarrow \mathbb{G}$ is faithful, the categorical crossed module is said to be a normal sub-categorical group of $\mathbb{G}$.

Let us recall from [10] that, given a categorical crossed module $(\mathbb{G}, \mathbb{A}, \varphi, T, \nu, \chi)$, we can construct the quotient categorical group given by the following diagram

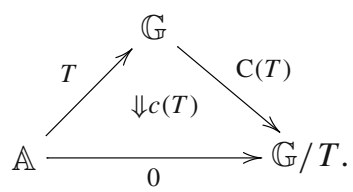

Its construction is the same as for the cokernel of a homomorphism in $\mathcal{S C G}$, the only difference is the tensor product of arrows: given two arrows $[A, f]: X \rightarrow \longrightarrow$ and $[B, g]: H \longrightarrow K$, their tensor product is given by the class of the prearrow with object part $A \otimes{ }^{Y} B$ and arrow part

$X \otimes H \stackrel{f \otimes g}{\longrightarrow} T A \otimes Y \otimes T B \otimes K \stackrel{1 \otimes v_{Y, B}^{-1} \otimes 1}{\longrightarrow} T A \otimes T\left({ }^{Y} B\right) \otimes Y \otimes K \simeq T\left(A \otimes{ }^{Y} B\right) \otimes Y \otimes K$ 
Homomorphisms of categorical crossed modules extend to the respective quotient categorical groups (see Lemma 7.6 in [13]) and the relation between kernels and quotients (Propositions 3.6 and 3.8 in [10]) is summarized in the following Proposition which states, on a categorical group level, the well known group theoretical facts that the underlying maps of crossed modules are proper maps and quotients are surjective.

Proposition 2.1 1. Let $(\varphi: \mathbb{G} \rightarrow \mathbb{E q}(\mathbb{A}), T: \mathbb{A} \rightarrow \mathbb{G}, v, \chi)$ be in $\mathcal{C C} M$ and consider the factorization through the kernel as in the following diagram

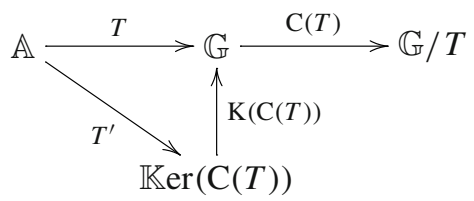

(a) $T^{\prime}$ is a full and essentially surjective homomorphism of $\mathbb{G}$-categorical crossed modules;

(b) if $T$ is faithful, then $\mathbb{A}$ and $\mathbb{K} \operatorname{er}(\mathrm{C}(T))$ are equivalent normal sub-categorical groups of $\mathbb{G}$.

2. Let $F: \mathbb{G} \rightarrow \mathbb{H}$ be in $\mathcal{C G}$ and consider the factorization through the quotient as in the following diagram

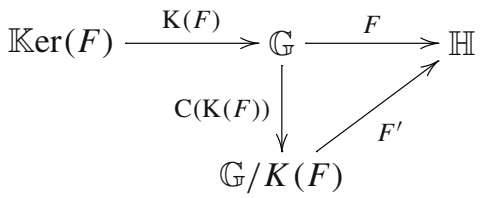

(a) $F^{\prime}$ is full and faithful;

(b) in particular, $F$ is a quotient (i.e., $F^{\prime}$ is an equivalence) if and only if $F$ is essentially surjective.

\section{Classifying singular extensions of categorical groups}

In this section we extend the results of [13] by giving a cohomological classification of the symmetric categorical group of singular extensions of a categorical group $\mathbb{G}$ by a given symmetric $\mathbb{G}$-categorical group $\mathbb{A}$. The variant here for the classification of all singular extensions uses, as a starting point, the simplicial set, $\operatorname{Ner}(\mathbb{G})$, nerve of a categorical group $\mathbb{G}$ introduced in [6].

Recall that Takeuchi-Ulbrich's cohomology (see [22,23]) allows to define cohomology groups of a simplicial set $K_{\bullet}$, with coefficients in a symmetric categorical group $\mathbb{A}$, by considering the cochain complex of symmetric categorical groups $\mathcal{C}\left(\mathbb{A}^{K} \bullet\right)$ that, in any dimension $n \geq 0$, consists of the functors from the discrete category $K_{n}$ to $\mathbb{A}$ (where tensor product, inverses and symmetry are given pointwise by the corresponding ones in $\mathbb{A}$ ) and where the coboundary homomorphisms are given by alternating sums of the coface homomorphisms $d_{i}^{*}: \mathbb{A}^{K_{n}} \longrightarrow \mathbb{A}^{K_{n+1}}$ induced by the face maps 
of $K_{\bullet}$. In particular, if $K_{\bullet}$ is the simplicial set $N e r(\mathbb{G})$, then 1-coboundaries and 2cocycles obtained from the cocomplex $\mathcal{C}\left(\mathbb{A}^{\operatorname{Ner}(\mathbb{G})}\right)$ are just (up to renumbering) those studied in [5]. If $\mathbb{A}$ is not merely a symmetric categorical group, but it is a symmetric $\mathbb{G}$-categorical group, the complex $\mathcal{C}\left(\mathbb{A}^{\text {Ner }(\mathbb{G})}\right)$ can be modified to a new one, $\mathcal{C}(\mathbb{G}, \mathbb{A})$, with the same symmetric categorical groups at any dimension, but where the coboundaries take into account the action of $\mathbb{G}$ on $\mathbb{A}$. We shall remark that Takeuchi-Ulbrich's cohomology of this complex would give cohomology groups $H^{n}(\operatorname{Ner}(\mathbb{G}), \mathbb{A})$ defined after passing to isomorphism classes of respective symmetric categorical groups of $n$-cocycles and $(n-1)$-coboundaries. However, our proposal of $\mathcal{H}^{2}$ (see Definition 1 ) is obtained by applying the notion of cokernel [24] to the coboundary homomorphism from the symmetric categorical group of 1-cochains to that one of 2-cocycles. In this way we obtain a symmetric categorical group whose $\pi_{0}$ (i.e., connected components) gives Takeuchi-Ulbrich's cohomology. Therefore, projection from $\mathcal{H}^{2}$ via $\pi_{0}$ produces, in several cases, other well-known cohomology groups (c.f. Example 3.4).

We start recalling, in a quick review, the study about general extensions of categorical groups with symmetric kernel done in [13].

\subsection{Singular extensions of categorical groups}

If $\mathbb{A}$ and $\mathbb{G}$ are categorical groups, with $\mathbb{A}$ symmetric, and $\varphi: \mathbb{G} \rightarrow \mathbb{E} \mathrm{q}_{s}(\mathbb{A})$ is a symmetric action, an extension of $\mathbb{G}$ by $\mathbb{A}$ is a 6-tuple

$$
\left(\mathbb{B}, \psi: \mathbb{B} \rightarrow \mathbb{E q}_{s}(\mathbb{A}), T: \mathbb{A} \rightarrow \mathbb{B}, \nu, \chi, S\right)
$$

where $(\mathbb{B}, \mathbb{A}, \psi, T, v, \chi)$ is an object in $\operatorname{SCCM}$ with $T$ faithful (that is, $\mathbb{A}$ is a symmetric normal sub-categorical group of $\mathbb{B}$ ) and $S: \mathbb{B} / T \rightarrow \mathbb{G}$ is an equivalence in $\mathcal{C G}$. When $\psi, v$ and $\chi$ are understood, we denote an extension of $\mathbb{G}$ by $\mathbb{A}$ by

$$
\mathbb{A} \stackrel{T}{\longrightarrow} \mathbb{B} \stackrel{\mathrm{C}(T)}{\longrightarrow} \mathbb{B} / T \stackrel{S}{\longrightarrow} \mathbb{G}
$$

Note that in any extension of $\mathbb{G}$ by $\mathbb{A}$ the symmetric categorical group $\mathbb{A}$ is equivalent, as a normal sub-categorical group, to the kernel of $S \cdot \mathrm{C}(T): \mathbb{B} \rightarrow \mathbb{B} / T \rightarrow \mathbb{G}$. Also note that, since $\mathbb{A}$ is symmetric, there is a symmetric action of $\mathbb{G}$ on $\mathbb{A}$ (that we recall as the action of $\mathbb{G}$ on $\mathbb{A}$ induced by the extension) given by the composite

$$
\mathbb{G} \stackrel{S^{-1}}{\longrightarrow} \mathbb{B} / T \stackrel{\tilde{\psi}}{\longrightarrow} \mathbb{E} \mathrm{q}_{s}(\mathbb{A})
$$

where $(\widetilde{\psi}, \widetilde{\delta})$ is the factorization through the quotient

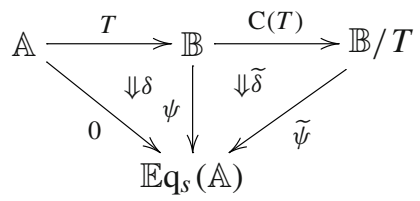


where the morphism $\delta: \psi \cdot T \Rightarrow 0$ is given by $\delta_{A, B}:{ }^{T A} B \rightarrow B$ defined by

$$
{ }^{T A} B \simeq{ }^{T A} B \otimes A \otimes A^{*} \stackrel{\chi_{A, B} \otimes 1}{\longrightarrow} A \otimes B \otimes A^{*} \stackrel{c_{A, B} \otimes 1}{\longrightarrow} B \otimes A \otimes A^{*} \simeq B
$$

Extensions of $\mathbb{G}$ by $\mathbb{A}$ are the objects of a 2-groupoid, $\mathbb{E x t}(\mathbb{G}, \mathbb{A})$, whose 1-cells are the homomorphisms of extensions, that is, 4-tuples

$$
(R, \lambda, \tau, m):(\mathbb{B}, \mathbb{A}, \psi, T, v, \chi, S) \rightarrow\left(\mathbb{B}^{\prime}, \mathbb{A}, \psi^{\prime}, T^{\prime}, v^{\prime}, \chi^{\prime}, S^{\prime}\right)
$$

where $m: S \Rightarrow S^{\prime} \cdot \widetilde{R}$ is a morphism in $\mathcal{C G}$ (with $\widetilde{R}$ induced between the quotient categorical groups by $\lambda$ and $\tau)$, and $\left(R, \operatorname{Id}_{\mathbb{A}}, \lambda, \tau\right):(\mathbb{B}, \mathbb{A}, \psi, T, \nu, \chi) \rightarrow$ $\left(\mathbb{B}^{\prime}, \mathbb{A}, \psi^{\prime}, T^{\prime}, v^{\prime}, \chi^{\prime},\right)$ is a homomorphism in $\operatorname{SCCM}$

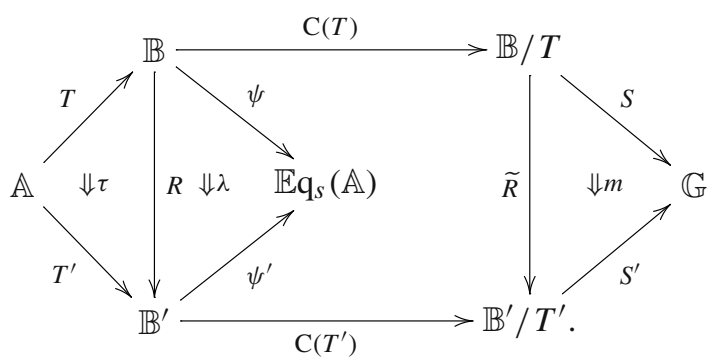

A morphism of extensions

$\beta:(R, \lambda, \tau, m) \Rightarrow\left(R^{\prime}, \lambda^{\prime}, \tau^{\prime}, m^{\prime}\right):(\mathbb{B}, \mathbb{A}, \psi, T, v, \chi, S) \rightarrow\left(\mathbb{B}^{\prime}, \mathbb{A}, \psi^{\prime}, T^{\prime}, v^{\prime}, \chi^{\prime}, S^{\prime}\right)$

is a morphism $(\beta, \mathrm{id}):\left(R, \operatorname{Id}_{\mathbb{A}}, \lambda, \tau\right) \Rightarrow\left(R^{\prime}, \operatorname{Id}_{\mathbb{A}}, \lambda^{\prime}, \tau^{\prime}\right)$ in $\mathcal{S C C} \mathcal{M}$ making compatible the morphisms $m$ and $m^{\prime}$ (see condition (ext1) in [13]).

Now we pay special attention to singular extensions of $\mathbb{G}$ by $\mathbb{A}$, that is, extensions such that the action of $\mathbb{G}$ on $\mathbb{A}$ induced by the extension coincides, up to a morphism, with the given one. More precisely, we have (see Definition 8.7 in [13]):

Definition 3.1 Let $\varphi: \mathbb{G} \rightarrow \mathbb{E} \mathrm{q}_{s}(\mathbb{A})$ be a symmetric action. A $\varphi$-extension of $\mathbb{G}$ by $\mathbb{A}$ (or a singular extension if $\varphi$ is understood) is a 7-tuple

$$
\left(\mathbb{B}, \psi: \mathbb{B} \rightarrow \mathbb{E q}_{s}(\mathbb{A}), T: \mathbb{A} \rightarrow \mathbb{B}, v, \chi, S, s\right)
$$

where

$$
\mathbb{A} \stackrel{T}{\longrightarrow} \mathbb{B} \stackrel{\mathrm{C}(T)}{\longrightarrow} \mathbb{B} / T \stackrel{S}{\longrightarrow} \mathbb{G}
$$

is an extension of $\mathbb{G}$ by $\mathbb{A}$, and 


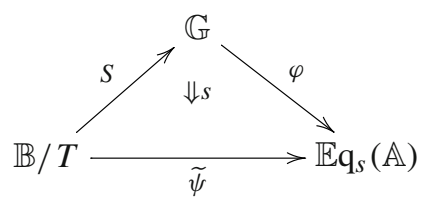

is a morphism in $\mathcal{C G}$.

A homomorphism of $\varphi$-extensions

$$
(R, \lambda, \tau, m):(\mathbb{B}, \mathbb{A}, \psi, T, v, \chi, S, s) \rightarrow\left(\mathbb{B}^{\prime}, \mathbb{A}, \psi^{\prime}, T^{\prime}, v^{\prime}, \chi^{\prime}, S^{\prime}, s^{\prime}\right)
$$

is a homomorphism of extensions $(R, \lambda, \tau, m)$ such that the following diagram commutes

$(\varphi \operatorname{ext} 1)$

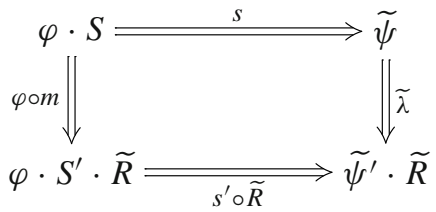

A morphism of $\varphi$-extensions

$$
\begin{aligned}
\beta:(R, \lambda, \tau, m) & \Rightarrow\left(R^{\prime}, \lambda^{\prime}, \tau^{\prime}, m^{\prime}\right):(\mathbb{B}, \mathbb{A}, \psi, T, v, \chi, S, s) \\
& \rightarrow\left(\mathbb{B}^{\prime}, \mathbb{A}, \psi^{\prime}, T^{\prime}, v^{\prime}, \chi^{\prime}, S^{\prime}, s^{\prime}\right)
\end{aligned}
$$

is just a morphism of extensions.

Note that the three notions in the above definition (singular extension, homomorphism and morphism) have equivalent formulations that, occasionally, can be easier to handle than the original ones (see Remark 8.8 in [13]).

It is remarkable the fact that $\varphi$-extensions of $\mathbb{G}$ by $\mathbb{A}$, with their homomorphisms and morphisms, form a 2-groupoid denoted $\mathbb{O} \operatorname{pext}(\varphi, \mathbb{G}, \mathbb{A})$ (see Remark 8.4 in [13]).

Remark 3.2 For any symmetric $\mathbb{G}$-categorical group $\mathbb{A}$, a notion of singular extension of $\mathbb{G}$ by $\mathbb{A}$ was introduced in $[9$, Definition 4.1] as a pair $(\mathcal{E}, \varphi)$ where $\mathcal{E}: \mathbb{A} \stackrel{j}{\longrightarrow}$ $\mathbb{E} \stackrel{P}{\longrightarrow} \mathbb{G}$ is a sequence of categorical group homomorphisms, $P$ is an essentially surjective fibration, $j$ gives an equivalence between $\mathbb{A}$ and the fibre category of $P$ over the unit object $I$ of $\mathbb{G}$, and

$$
\varphi=\left(\varphi_{E, A}: E \otimes j(A) \longrightarrow j\left({ }^{p(E)} A\right) \otimes E\right)_{(A, E) \in \operatorname{Obj}(\mathbb{A}) \times \operatorname{Obj}(\mathbb{E})}
$$

is a family of natural isomorphisms in $\mathbb{E}$ that have to satisfy suitable coherence conditions. This notion is equivalent (c.f. [20]) to the one considering essentially surjective homomorphisms and their homotopy kernels and then, according to Proposition 2.1, to the notion of singular extension here considered. These different approaches are, in fact, the categorical-group version of the group case, where an extension can be 
presented as a surjective homomorphism together with its kernel or as a normal subgroup together with its cokernel. The advantage with the definition that we handle in this paper, is that, when the normal sub-categorical group is symmetric, it is easy to construct an action of the quotient on the kernel, much easier than doing the same if one defines an extension as an essentially surjective homomorphism with a symmetric kernel. The price to pay is that (contrarily to what happens with groups) to be a normal sub-categorical group is a structure, and not a property, and the only reasonable way to handle such a structure is to see it as a special case of the general structure of categorical crossed module.

\subsection{The second cohomology categorical group}

We fix a categorical group $\mathbb{G}$ and a symmetric $\mathbb{G}$-categorical group $\mathbb{A}=(\mathbb{A}, \varphi: \mathbb{G} \rightarrow$ $\left.\mathbb{E} \mathrm{q}_{s}(\mathbb{A})\right)$ and, since the action $\varphi$ has been fixed, here after we usually avoid to name it. Below we describe explicitly the cochain complex of symmetric categorical groups $\mathcal{C}(\mathbb{G}, \mathbb{A})$ in low dimensions.

$-\mathcal{C}^{0}(\mathbb{G}, \mathbb{A})$ is the trivial category.

$-\mathcal{C}^{1}(\mathbb{G}, \mathbb{A})$ : Its objects are maps $\gamma: \operatorname{Obj}(\mathbb{G}) \rightarrow \operatorname{Obj}(\mathbb{A})$; an arrow $\gamma \rightarrow \gamma^{\prime}$ is a family $\left\{\gamma_{X} \rightarrow \gamma_{X}^{\prime} \mid X \in \operatorname{Obj}(\mathbb{G})\right\}$ of morphisms in $\mathbb{A}$.

$-\mathcal{C}^{2}(\mathbb{G}, \mathbb{A})$ : Its objects are maps $f \mapsto p(f)$ from the set of morphisms of $\mathbb{G}$, of the form $f: X \otimes Y \rightarrow Z$, into the objects of $\mathbb{A}$; an arrow $p \rightarrow p^{\prime}$ is a family $\left\{p(f) \rightarrow p^{\prime}(f)\right\}_{f}$ of morphisms in $\mathbb{A}$ indexed by morphisms in $\mathbb{G}$ of the form $f: X \otimes Y \rightarrow Z$.

$-\mathcal{C}^{3}(\mathbb{G}, \mathbb{A})$ : Its objects are maps $\alpha \mapsto q(\alpha)$ from the set of commutative diagrams $\alpha$ in $\mathbb{G}$ of the form

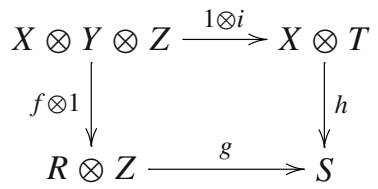

(where the associativity isomorphisms are omitted) into the objects of $\mathbb{A}$; an arrow $q \rightarrow q^{\prime}$ is a family $\left\{q(\alpha) \rightarrow q^{\prime}(\alpha)\right\}_{\alpha}$ of morphisms in $\mathbb{A}$ indexed by commutative squares $\alpha$ as above.

$-\mathcal{C}^{4}(\mathbb{G}, \mathbb{A})$ : Its objects are maps $\theta \mapsto r(\theta)$ from the set of commutative diagrams $\theta$ in $\mathbb{G}$ of the form

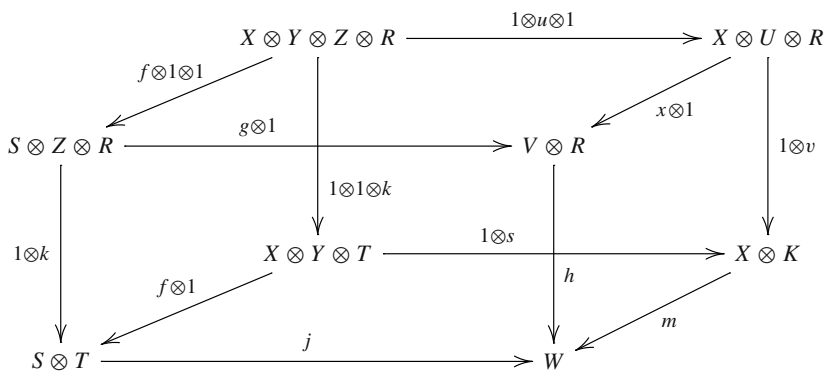


(where the associativity isomorphisms are omitted) into the objects of $\mathbb{A}$; an arrow $r \rightarrow r^{\prime}$ is a family $\left\{r(\theta) \rightarrow r^{\prime}(\theta)\right\}_{\theta}$ of morphisms in $\mathbb{A}$ indexed for commutative cubes $\theta$ as above.

The corresponding coboundaries are defined by:

- $\partial^{0}(*)(X)=I$ for all $X \in \mathbb{G}$;

$-\partial^{1}(\gamma)(f)={ }^{X} \gamma_{Y} \otimes \gamma_{X} \otimes \gamma_{Z}^{*}$ for all $f: X \otimes Y \rightarrow Z$ in $\mathbb{G}$;

$-\partial^{2}(p)(\alpha)=p(f) \otimes p(h)^{*} \otimes p(g) \otimes{ }^{X} p(i)^{*}$ for all diagrams $\alpha$ as in (1);

$-\partial^{3}(q)(\theta)=q\left(\alpha_{0}\right) \otimes q\left(\alpha_{1}\right)^{*} \otimes q\left(\alpha_{2}\right) \otimes q\left(\alpha_{3}\right)^{*} \otimes X_{q}\left(\alpha_{4}\right)$ for all diagrams $\theta$ as in

(2) where $\alpha_{0} \otimes 1$ is its upper face, $\alpha_{1}$ its down face, $\alpha_{3}$ its front face, $1 \otimes \alpha_{4}$ its back face and $\alpha_{2}$ its right side face.

From the truncated cocomplex

$$
\mathcal{C}^{0}(\mathbb{G}, \mathbb{A}) \stackrel{\partial^{0}}{\longrightarrow} \mathcal{C}^{1}(\mathbb{G}, \mathbb{A}) \stackrel{\partial^{1}}{\longrightarrow} \mathcal{C}^{2}(\mathbb{G}, \mathbb{A}) \stackrel{\partial^{2}}{\longrightarrow} \mathcal{C}^{3}(\mathbb{G}, \mathbb{A}) \stackrel{\partial^{3}}{\longrightarrow} \mathcal{C}^{4}(\mathbb{G}, \mathbb{A})
$$

(adding the normalization condition $\gamma_{I} \cong I$ compatible with arrows $\gamma \rightarrow \gamma^{\prime}$ ) we get the cohomology categorical group $\mathcal{H}^{2}(\mathbb{G}, \mathbb{A})$ following the general construction given in [11, Definition 3.1]: take first the relative kernel $\mathcal{Z}^{2}(\mathbb{G}, \mathbb{A})$ of $\left(\partial^{2}, \partial^{3}\right)$, then $\mathcal{H}^{2}(\mathbb{G}, \mathbb{A})$ is the cokernel of the factorization $\mathcal{T}$ of $\partial^{1}$ through $\mathcal{Z}^{2}(\mathbb{G}, \mathbb{A})$.

For the reader's convenience, we make now such a construction explicit.

We start describing the categorical group $\mathcal{Z}^{2}(\mathbb{G}, \mathbb{A})$ of normalized 2-cocycles of $\mathbb{G}$ with coefficients in $\mathbb{A}$. Note that its set of objects is just (up to a dimensional shift) the set of normalized 1-cocycles of $\mathbb{G}$ with coefficients in the $\mathbb{G}$-module $\mathbb{A}$ introduced in [9].

- The objects of $\mathcal{Z}^{2}(\mathbb{G}, \mathbb{A})$ are systems $(t, p)$, consisting of the following data:

(a) For any three objects $X, Y, Z$ and any morphism $f: X \otimes Y \rightarrow Z$ of $\mathbb{G}$, there is an object $p(f) \in \mathbb{A}$.

(b) For any four morphisms

$$
f: X \otimes Y \rightarrow R, g: R \otimes Z \rightarrow S, h: X \otimes T \rightarrow S, i: Y \otimes Z \rightarrow T
$$

in $\mathbb{G}$ making diagram (1) commutative, there is a morphism

$$
t_{f, g, h, i}: p(f) \otimes p(g) \rightarrow{ }^{X} p(i) \otimes p(h)
$$

in $\mathbb{A}$ subject to the following conditions:

(NC) Normalization conditions:

NC1: For any object $X$ in $\mathbb{G}, p\left(r_{X}\right) \cong I \cong p\left(l_{X}\right)$;

NC2: For any $f: X \otimes Y \rightarrow Z, t_{r, f, f, l}: I \otimes p(f) \rightarrow{ }^{X} I \otimes p(f)$ is the canonical isomorphism.

(CC) Cocycle condition: For any ten objects $X, Y, Z, R, S, T, U, V, W, K$ and ten morphisms 
$f: X \otimes Y \rightarrow S, g: S \otimes Z \rightarrow V, h: V \otimes R \rightarrow W, j: S \otimes T \rightarrow W, k: Z \otimes R \rightarrow T$

$m: X \otimes K \rightarrow W, s: Y \otimes T \rightarrow K, x: X \otimes U \rightarrow V, u: Y \otimes Z \rightarrow U, v: U \otimes R \rightarrow K$

in $\mathbb{G}$ making diagram (2) commutative, the diagram

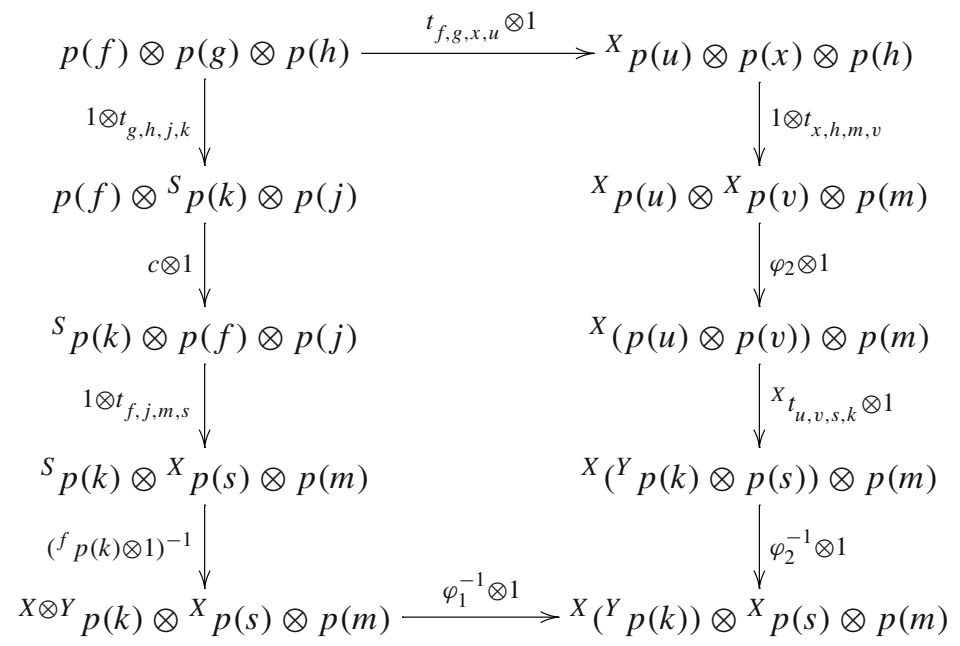

commutes.

- An arrow $\alpha:(t, p) \rightarrow\left(t^{\prime}, p^{\prime}\right)$ in $\mathcal{Z}^{2}(\mathbb{G}, \mathbb{A})$ is a family $\left\{\alpha_{f}: p(f) \rightarrow p^{\prime}(f)\right\}_{f}$ of morphisms in $\mathbb{A}$ indexed by morphisms $f: X \otimes Y \rightarrow Z$ in $\mathbb{G}$, such that for any commutative diagram like (1), the following diagram commutes

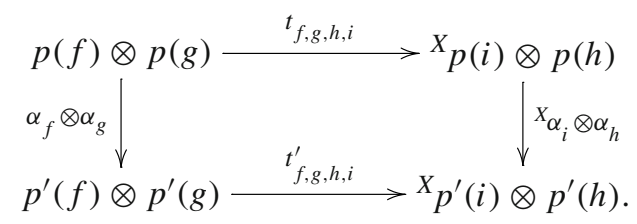

- The tensor product in $\mathcal{Z}^{2}(\mathbb{G}, \mathbb{A})$ is given, on objects, by

$$
(t, p) \otimes\left(t^{\prime}, p^{\prime}\right)=\left(t \otimes t^{\prime}, p \otimes p^{\prime}\right)
$$

where, for any $f: X \otimes Y \rightarrow Z,\left(p \otimes p^{\prime}\right)(f)=p(f) \otimes p^{\prime}(f)$ and, for any commutative diagram like $(1),\left(t \otimes t^{\prime}\right)_{f, g, h, i}$ is the dotted arrow making the following diagram commutative 


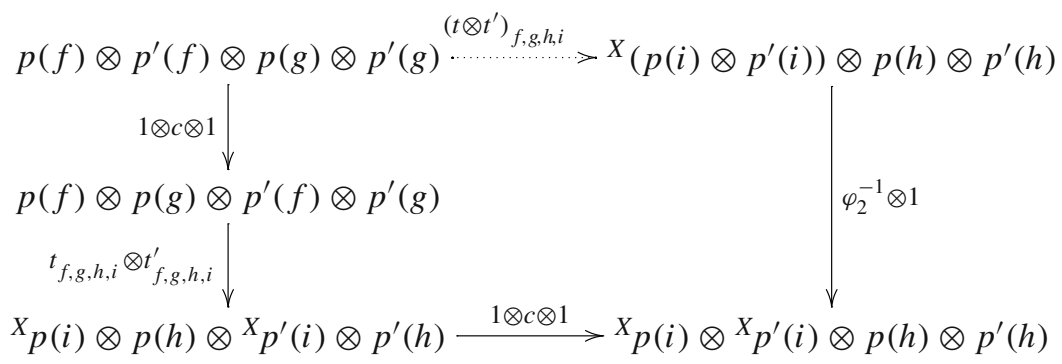

The tensor product on arrows is pointwise. The rest of the symmetric categorical group structure of $\mathcal{Z}^{2}(\mathbb{G}, \mathbb{A})$ is induced by that of $\mathbb{A}$.

Remark 3.3 To help the reader with this notion of cocycle, let us look at what happens when $\mathbb{G}$ and $\mathbb{A}$ are discrete (see also Example 3.4). If $\mathbb{G}$ is discrete, to have an arrow $f: X \otimes Y \rightarrow Z$ just means that $Z=X \otimes Y$, so that the component $p$ of a cocycle $(t, p)$ amounts to a map

$$
p: \operatorname{Obj}(\mathbb{G}) \times \operatorname{Obj}(\mathbb{G}) \rightarrow \operatorname{Obj}(\mathbb{A}), \quad X, Y \mapsto p(X, Y)
$$

Moreover, to give a commutative diagram like (1) means that

$$
X \otimes Y=R, \quad R \otimes Z=S, \quad X \otimes T=S, \quad Y \otimes Z=T .
$$

Therefore,

$$
\begin{aligned}
& p(f)=p(X, Y), \quad p(g)=p(R, Z)=p(X \otimes Y, Z) \\
& p(i)=p(Y, Z), \quad p(h)=p(X, T)=p(X, Y \otimes Z)
\end{aligned}
$$

Finally, the existence of a morphism $t_{f, g, h, i}: p(f) \otimes p(g) \rightarrow{ }^{X} p(i) \otimes p(h)$ means that the usual cocycle equation holds:

$$
p(X, Y) \otimes p(X \otimes Y, Z)={ }^{X} p(Y, Z) \otimes p(X, Y \otimes Z) .
$$

The homomorphism of symmetric categorical groups

$$
\mathcal{T}=\left(\mathcal{T}, \mathcal{T}_{2}\right): \mathcal{C}^{1}(\mathbb{G}, \mathbb{A}) \rightarrow \mathcal{Z}^{2}(\mathbb{G}, \mathbb{A})
$$

can be described as follows. Given an object $\gamma \in \mathcal{C}^{1}(\mathbb{G}, \mathbb{A}), \mathcal{T}(\gamma)=\left(t^{\gamma}, p^{\gamma}\right)$ where, for any $f: X \otimes Y \rightarrow Z, p^{\gamma}(f)={ }^{X} \gamma_{Y} \otimes \gamma_{X} \otimes \gamma_{Z}^{*}$ and, for any diagram like (1), 
$t_{f, g, h, i}^{\gamma}$ is the dotted arrow making the following diagram commutative

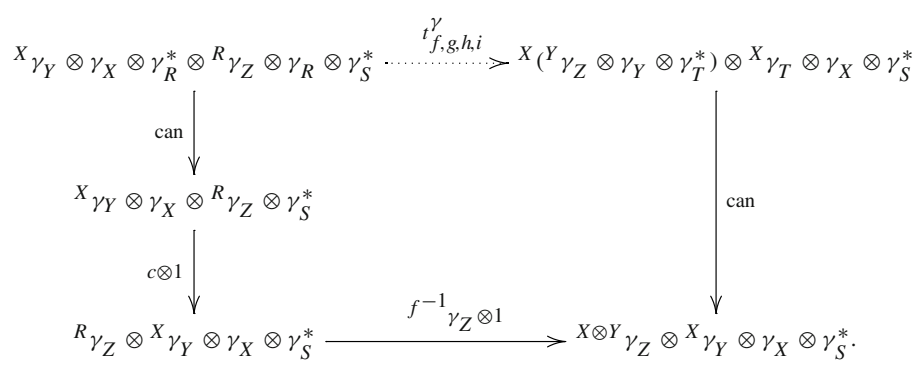

On arrows $\chi: \gamma \rightarrow \gamma^{\prime}, \mathcal{T}(\chi):\left(t^{\gamma}, p^{\gamma}\right) \rightarrow\left(t^{\prime \gamma}, p^{\prime \gamma}\right)$ is given, for any $f: X \otimes Y \rightarrow$ $Z$, by $T(\chi)_{f}={ }^{X} \chi_{Y} \otimes \chi_{X} \otimes \chi_{Z}^{*}: p^{\gamma}(f) \rightarrow p^{\prime \gamma}(f)$. The monoidal structure

$$
\mathcal{T}_{2}: \mathcal{T}(\gamma) \otimes \mathcal{T}\left(\gamma^{\prime}\right) \rightarrow \mathcal{T}\left(\gamma \otimes \gamma^{\prime}\right)
$$

is the canonical isomorphism

$$
\left.X_{\gamma_{Y}} \otimes \gamma_{X} \otimes \gamma_{Z}^{*} \otimes{ }^{X} \gamma_{Y}^{\prime} \otimes \gamma_{X}^{\prime} \otimes \gamma_{Z}^{\prime *} \stackrel{\text { can }}{\longrightarrow} X_{\left(\gamma_{Y}\right.} \otimes \gamma_{Y}^{\prime}\right) \otimes \gamma_{X} \otimes \gamma_{X}^{\prime} \otimes \gamma_{Z}^{*} \otimes \gamma_{Z}^{\prime *}
$$

obtained from the symmetry of $\mathbb{A}$ and the isomorphism $\varphi$ of the action of $\mathbb{G}$ on $\mathbb{A}$.

We can close the previous discussion with the following:

Definition 1 The second cohomology categorical group of $\mathbb{G}$ with coefficients in the symmetric $\mathbb{G}$-categorical group $\mathbb{A}, \mathcal{H}^{2}(\mathbb{G}, \mathbb{A})$, is the cokernel of the homomorphism of symmetric categorical groups $\mathcal{T}: \mathcal{C}^{1}(\mathbb{G}, \mathbb{A}) \rightarrow \mathcal{Z}^{2}(\mathbb{G}, \mathbb{A})$, that is

$$
\mathcal{H}^{2}(\mathbb{G}, \mathbb{A})=\operatorname{Coker}(\mathcal{T})
$$

The following example shows that our $\mathcal{H}^{2}(\mathbb{G}, \mathbb{A})$ particularizes, in the discrete case, to several well-known cohomology groups.

Example 3.4 1. If $\mathbb{G}$ is the discrete categorical group $[G]_{0}$ associated with a group $G$, then the set of objects of $\mathcal{Z}^{2}\left([G]_{0}, \mathbb{A}\right)$ is identified with the set of Ulbrich's 3 -cocycles of $G$ with coefficients in $\mathbb{A}$, (see [23]). In particular (see Example 3 in [9]), if $\mathbb{A}$ is the discrete symmetric $[G]_{0}$-categorical group $[A]_{0}$ associated with a $G$-module $A$, then $\mathcal{Z}^{2}\left([G]_{0},[A]_{0}\right)$ is the discrete symmetric categorical group $\left[\mathrm{Z}^{2}(G, A)\right]_{0}$ associated with the abelian group of Eilenberg-Mac Lane 2-cocycles of $G$ with coefficients in $A$.

2. If $\mathbb{G}=[G]_{0}$ and, moreover, $\mathbb{A}=[A]_{1}$ is the symmetric $[G]_{0}$-categorical group with only one object associated with a $G$-module $A$, then $\mathcal{Z}^{2}\left([G]_{0},[A]_{1}\right)$ is the discrete symmetric categorical group associated with the abelian group of Eilenberg-Mac Lane 3-cocycles of $G$ with coefficients in the $G$-module $A$. 
3. When the action of $\mathbb{G}$ on $\mathbb{A}$ is trivial, then the set of objects of $\mathcal{Z}^{2}(\mathbb{G}, \mathbb{A})$ is the set of 1-cocycles introduced in [6]. If, moreover, $\mathbb{G}=[G]_{0}$, then the set of objects of $\mathcal{Z}^{2}\left([G]_{0}, \mathbb{A}\right)$ coincides with the set of Breen's 1-cocycles introduced in [3].

In conclusion, the cohomology groups introduced by Eilenberg-Mac Lane [19], Ulbrich [23], Breen [3], and Carrasco and Cegarra [6], can be obtained as special instances of our symmetric categorical group $\mathcal{H}^{2}(\mathbb{G}, \mathbb{A})$ via the functor of connected components $\pi_{0}$.

\subsection{The classification theorem}

In order to compare $\mathcal{H}^{2}(\mathbb{G}, \mathbb{A})$ with the 2-groupoid $\mathbb{O} \operatorname{pext}(\varphi, \mathbb{G}, \mathbb{A})$, in the next theorem we look at $\mathcal{H}^{2}(\mathbb{G}, \mathbb{A})$ as a monoidal bicategory (this fact for the cokernel of any homomorphism in $\mathcal{S C G}$ has been already remarked in Sect. 2). Following the same line of arguments than that carried out in Proposition 9.1 and Theorem 9.3 in [13], we will show a biequivalence between $\mathcal{H}^{2}(\mathbb{G}, \mathbb{A})$ and $\mathbb{O} \operatorname{pext}(\varphi, \mathbb{G}, \mathbb{A})$ by giving only the constructive part of the proof and will omit the long diagrammatic arguments needed to check that the various constructions fulfill the requested coherence conditions. As we will remark after its proof, the next theorem extends, to the appropriate bicategorical context, the classification of singular extensions of categorical groups given in [9].

Theorem 3.5 Let $\mathbb{G}$ be a categorical group and $\varphi: \mathbb{G} \rightarrow \mathbb{E} \mathrm{q}_{s}(\mathbb{A})$ a symmetric action. Then, there exists a biequivalence of bicategories

$$
\overline{\mathcal{E}}: \mathcal{H}^{2}(\mathbb{G}, \mathbb{A}) \rightarrow \mathbb{O} \operatorname{pext}(\varphi, \mathbb{G}, \mathbb{A})
$$

Proof We split the proof in four steps.

Step 1 We construct a 2-functor

$$
\mathcal{E}: \mathcal{Z}^{2}(\mathbb{G}, \mathbb{A}) \rightarrow \mathbb{O} \operatorname{pext}(\varphi, \mathbb{G}, \mathbb{A})
$$

where $\mathcal{Z}^{2}(\mathbb{G}, \mathbb{A})$ is seen as a 2-category with only identity 2-arrows.

Let $(t, p)$ be a normalized 2-cocycle of $\mathbb{G}$ with coefficients in $\mathbb{A}$ and consider the categorical group $\mathbb{A} \times{ }_{(t, p)} \mathbb{G}$ described below:

- Objects: $\operatorname{Obj}\left(\mathbb{A} \times{ }_{(t, p)} \mathbb{G}\right)=\operatorname{Obj}(\mathbb{A}) \times \operatorname{Obj}(\mathbb{G})$.

- Morphisms: Pairs $(u, f):(A, X) \rightarrow(B, Y)$ where $f: X \rightarrow Y$ is a morphism in $\mathbb{G}$ and $u: A \otimes p\left(f l_{X}\right) \rightarrow B$ is a morphism in $\mathbb{A}$.

- Identities: $1_{(A, X)}=\left(A \otimes p\left(l_{X}\right) \rightarrow A \otimes I \stackrel{r_{A}}{\longrightarrow} A, 1_{X}\right)$.

- Composition: Given morphisms $(A, X) \stackrel{(u, f)}{\longrightarrow}(B, Y) \stackrel{(v, g)}{\longrightarrow}(C, Z)$, their composition is $(w, g f):(A, X) \rightarrow(C, Z)$ where $w: A \otimes p\left(g f l_{X}\right) \rightarrow C$ is the 
morphism in $\mathbb{A}$ determined by the commutativity of the following diagram:

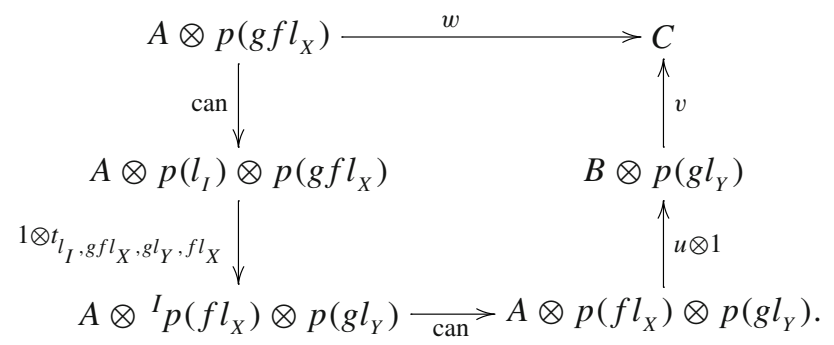

- Tensor product: For objects it is given by

$$
(A, X) \otimes(B, Y)=\left(A \otimes{ }^{X} B \otimes p\left(1_{X \otimes Y}\right), X \otimes Y\right)
$$

and for arrows $(u, f):(A, X) \rightarrow(C, Z)$ and $(v, g):(B, Y) \rightarrow(D, W)$ it is given by

$$
\begin{aligned}
(u, f) \otimes(v, g) & =(w, f \otimes g):\left(A \otimes{ }^{X} B \otimes p\left(1_{X \otimes Y}\right), X \otimes Y\right) \\
& \longrightarrow\left(C \otimes{ }^{Z} D \otimes p\left(1_{Z \otimes W}\right), Z \otimes W\right)
\end{aligned}
$$

where $w$ is the morphism making commutative the following diagram:

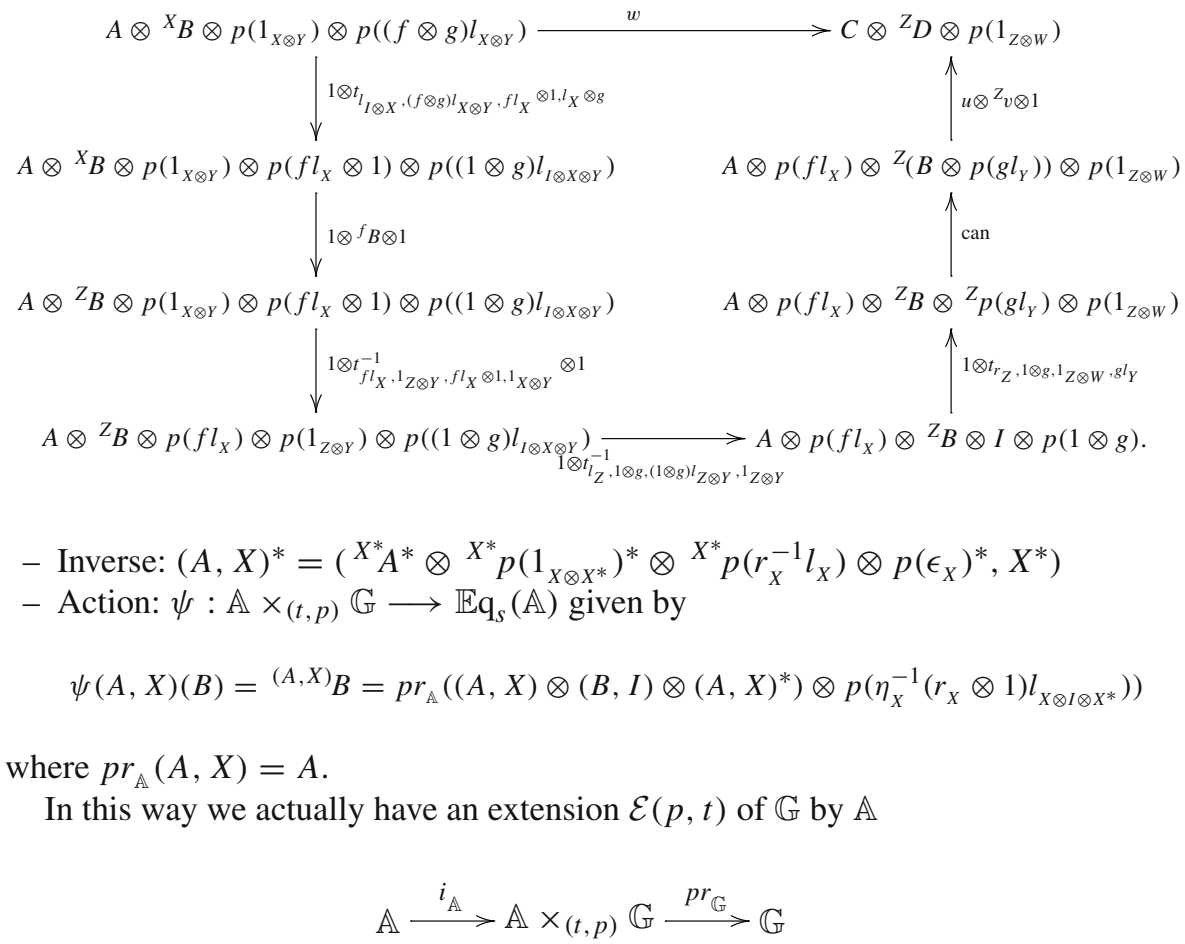


where $i_{\mathbb{A}}(A, X)=A$ is a faithful homomorphism and $p r_{\mathbb{G}}(A, X)=X$ is an essentially surjective homomorphism of categorical groups. Moreover, there is an equivalence between $\mathbb{A}$ and $\mathbb{K} \operatorname{er}\left(p r_{\mathbb{G}}\right)$ given as follows: If $(A, X) \in \mathbb{K} \operatorname{er}\left(p r_{\mathbb{G}}\right)$, there exists an arrow $a: X \rightarrow I$ in $\mathbb{G}$ and then $\Gamma: \mathbb{K e r}\left(p r_{\mathbb{G}}\right) \longrightarrow \mathbb{A}$ is given, on objects, by $\Gamma(A, X)=$ $A \otimes p\left(a r_{X}\right)$ and, on arrows $(u, f):(A, X) \rightarrow(B, Y), \Gamma(u, f): A \otimes p\left(a r_{X}\right) \longrightarrow$ $B \otimes p\left(b r_{Y}\right)$ is the morphism determined, in a canonical way, by $u: A \otimes p\left(f l_{X}\right) \rightarrow B$ and $t_{f l_{X}, b r_{Y}, l_{I}, a r_{X}}: p\left(f l_{X}\right) \otimes p\left(b r_{Y}\right) \longrightarrow{ }^{I} p\left(a r_{X}\right) \otimes p\left(l_{I}\right)$. Also (recall Proposition 2.1) there is an equivalence $(\mathbb{A} \times(t, p) \mathbb{G}) / i_{\mathbb{A}} \simeq \mathbb{G}$ : For any arrow from $(A, X)$ to $(B, Y)$ in $\mathbb{A} \times_{(t, p)} \mathbb{G}$ there is a morphism in $\mathbb{G}, X \rightarrow I \otimes Y$, which canonically determines the image $f: X \rightarrow Y$ through the equivalence.

The extension $\mathcal{E}(p, t)$ is actually a $\varphi$-extension of $\mathbb{G}$ by $\mathbb{A}$ because there is a morphism $\sigma: \varphi \cdot p r_{\mathbb{G}} \Rightarrow \widetilde{\psi}$ in $\mathcal{C G}$ obtained as follows. For any object $(A, X) \in \mathbb{A} \times(t, p) \mathbb{G}$, the morphism in $\mathbb{A} \sigma_{(A, X)}(B):{ }^{X} B \rightarrow \widetilde{\psi}(A, X)(B)={ }^{(A, X)} B$ is the part in $\mathbb{A}$ of the morphism in $\mathbb{A} \times{ }_{(t, p)} \mathbb{G},\left({ }^{X} B, I\right) \longrightarrow(A, X) \otimes(B, I) \otimes(A, X)^{*}$, canonically deduced from the following one $(A, X) \otimes(B, I) \longrightarrow\left({ }^{X} B, I\right) \otimes(A, X)$. This is given by the pair $\left(u, l_{X}^{-1} r_{X}\right)$ where $u: A \otimes{ }^{X} B \otimes p\left(1_{X \otimes I}\right) \otimes p\left(l_{X}^{-1} r_{X} l_{X \otimes I}\right) \longrightarrow{ }^{X} B \otimes A \otimes p\left(1_{I \otimes X}\right)$ is the morphism in $\mathbb{A}$ deduced from $t_{1, r, l^{-1} r l, 1}$ which is associated with the following commutative diagram

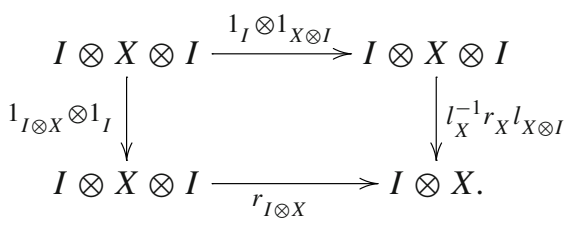

Let now $\alpha:(t, p) \rightarrow\left(t^{\prime}, p^{\prime}\right)$ be a morphism of 2-cocycles. Thus we have a family of morphisms in $\mathbb{A},\left\{\alpha_{f}: p(f) \rightarrow p^{\prime}(f)\right\}_{f: X \otimes Y \rightarrow Z}$ and we get a homomorphism of extensions $\mathcal{E}(\alpha): \mathcal{E}(t, p) \Rightarrow \mathcal{E}\left(t^{\prime}, p^{\prime}\right)$ according to the following diagrams
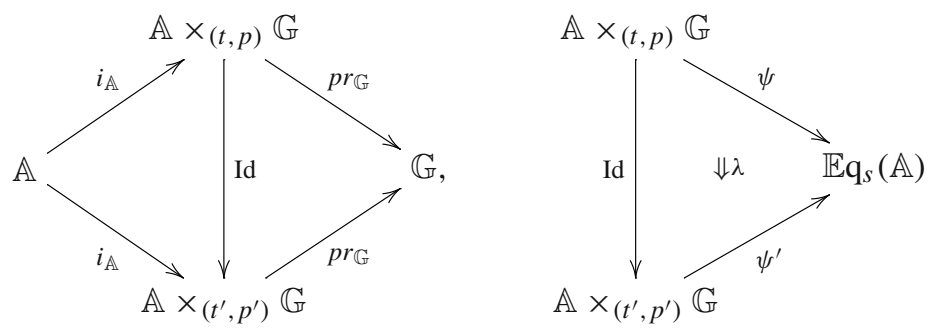

where Id: $\mathbb{A} \times_{(t, p)} \mathbb{G} \rightarrow \mathbb{A} \times_{\left(t^{\prime}, p^{\prime}\right)} \mathbb{G}$ is the identity functor with monoidal structure given by

$$
(A, X) \otimes_{(t, p)}(B, Y) \stackrel{\left(1 \otimes \alpha_{1_{X \otimes Y}}, 1\right)}{\longrightarrow}(A, X) \otimes_{\left(t^{\prime}, p^{\prime}\right)}(B, Y)
$$


and $\lambda_{(A, X), B}$ is determined by the identity in $\left.p r_{\mathbb{A}}\left((A, X) \otimes(B, I) \otimes(A, X)^{*}\right)\right)$ and $\alpha_{\eta_{X}^{-1}\left(r_{X} \otimes 1\right) l}: p\left(\eta_{X}^{-1}\left(r_{X} \otimes 1\right) l_{X \otimes I \otimes X^{*}}\right) \longrightarrow p^{\prime}\left(\eta_{X}^{-1}\left(r_{X} \otimes 1\right) l_{X \otimes I \otimes X^{*}}\right)$.

That $\mathcal{E}(\alpha)$ is a homomorphism in $\mathbb{O} \operatorname{pext}(\varphi, \mathbb{G}, \mathbb{A})$, that is, that condition ( $\varphi \operatorname{ext} 1)$ holds, follows from the own definition of $\lambda$.

Step 2 We construct now a 2-natural transformation

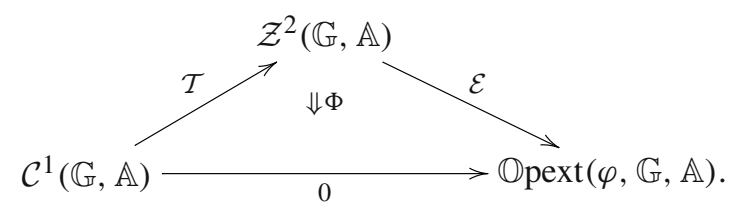

Let $\gamma: \operatorname{Obj}(\mathbb{G}) \rightarrow \operatorname{Obj}(\mathbb{A})$ a normalized object of $\mathcal{C}^{1}(\mathbb{G}, \mathbb{A})$. Then $\mathcal{T}(\gamma)=\left(t^{\gamma}, p^{\gamma}\right)$ and we get a homomorphism of extensions as follows
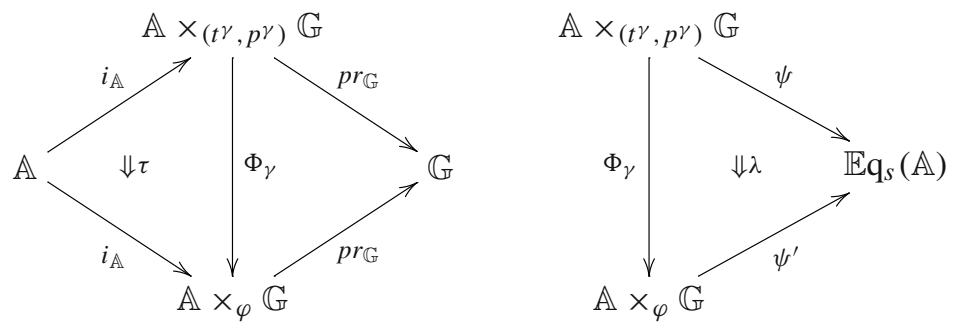

where $\mathbb{A} \times_{\varphi} \mathbb{G}$ is the semidirect product (see $[12,17]$ ) and the symmetric action

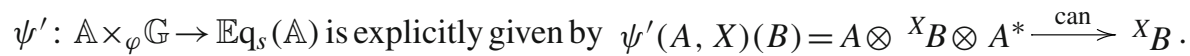
The functor $\Phi_{\gamma}$ is defined on objects by $\Phi_{\gamma}(A, X)=\left(A \otimes \gamma_{X}, X\right)$. Now, for any arrow $(u, f):(A, X) \rightarrow(B, Y)$ in $\left.\mathbb{A} \times{ }_{(t \gamma}, p^{\gamma}\right) \mathbb{G}$ we have arrows $f: X \rightarrow Y$ in $\mathbb{G}$ and $u: A \otimes p^{\gamma}\left(f l_{X}\right) \rightarrow B$ in $\mathbb{A}$ and since, by definition, $p^{\gamma}\left(f l_{X}\right) \cong \gamma_{X} \otimes \gamma_{Y}^{*}$, the arrow $u$ induces, in a canonical way, the required one $\Phi_{\gamma}(u, f):\left(A \otimes \gamma_{X}, X\right) \rightarrow\left(B \otimes \gamma_{Y}, Y\right)$. The monoidal structure of $\Phi_{\gamma}$ is obtained in a canonical way since

$$
\begin{aligned}
\Phi_{\gamma}((A, X) \otimes(B, Y)) & =\Phi_{\gamma}\left(A \otimes{ }^{X} B \otimes p^{\gamma}\left(1_{X \otimes Y}\right), X \otimes Y\right) \\
& =\left(A \otimes{ }^{X} B \otimes{ }^{X} \gamma_{Y} \otimes \gamma_{X} \otimes \gamma_{X \otimes Y}^{*} \otimes \gamma_{X \otimes Y}, X \otimes Y\right)
\end{aligned}
$$

whereas

$$
\begin{aligned}
\Phi_{\gamma}(A, X) \otimes \Phi_{\gamma}(B, Y) & =\left(A \otimes \gamma_{X}, X\right) \otimes\left(B \otimes \gamma_{Y}, Y\right) \\
& \cong\left(A \otimes \gamma_{X} \otimes{ }^{X} B \otimes{ }^{X} \gamma_{Y}, X \otimes Y\right) .
\end{aligned}
$$

Finally, since $\gamma_{I} \cong I$, the morphism $\tau: \Phi_{\gamma} \cdot i_{\mathbb{A}} \Rightarrow i_{\mathbb{A}}$ is defined in a canonical way and the morphism $\lambda: \psi \Rightarrow \psi^{\prime} \cdot \Phi_{\gamma}$ is the opposite of the morphism $\sigma$ used in Step 1. 
Step 3 Now we extend the 2-functor $\mathcal{E}: \mathcal{Z}^{2}(\mathbb{G}, \mathbb{A}) \rightarrow \mathbb{O} \operatorname{pext}(\varphi, \mathbb{G}, \mathbb{A})$, constructed in Step 1, to a homomorphism of bicategories

$$
\overline{\mathcal{E}}: \mathcal{H}^{2}(\mathbb{G}, \mathbb{A}) \rightarrow \mathbb{O} \operatorname{pext}(\varphi, \mathbb{G}, \mathbb{A})
$$

(we limit ourselves to define $\overline{\mathcal{E}}$ on objects, 1-arrows and 2-arrows; to check that $\overline{\mathcal{E}}$ is indeed a homomorphism of bicategories is long but essentially straightforward). On objects, $\overline{\mathcal{E}}$ is defined as $\mathcal{E}$. Consider now a 1-arrow $(\gamma, \alpha):(t, p) \rightarrow\left(t^{\prime}, p^{\prime}\right)$ in $\mathcal{H}^{2}(\mathbb{G}, \mathbb{A})$, that is, $\gamma \in \mathcal{C}^{1}(\mathbb{G}, \mathbb{A})$ and $\alpha:(t, p) \otimes\left(t^{\gamma}, p^{\gamma}\right) \rightarrow\left(t^{\prime}, p^{\prime}\right)$ is a $1-$ arrow in $\mathcal{Z}^{2}(\mathbb{G}, \mathbb{A})$; the homomorphism $\overline{\mathcal{E}}(\gamma, \alpha): \mathcal{E}(t, p) \rightarrow \mathcal{E}\left(t^{\prime}, p^{\prime}\right)$ factors through $\mathcal{E}\left((t, p) \otimes\left(t^{\gamma}, p^{\gamma}\right)\right)$ and is described in the following diagram, where $\Phi_{\gamma}$ and $\tau$ are as in Step 2, and Id is the identity functor with monoidal structure determined by $\alpha$ as in Step 1

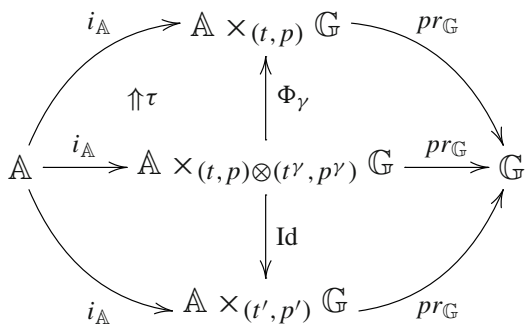

Consider now a 2-arrow $\beta:(\gamma, \alpha) \Rightarrow\left(\gamma^{\prime}, \alpha^{\prime}\right)$ in $\mathcal{H}^{2}(\mathbb{G}, \mathbb{A})$, that is, a morphism $\beta: \gamma \Rightarrow \gamma^{\prime}$ in $\mathcal{C}^{1}(\mathbb{G}, \mathbb{A})$ (i.e., a family of morphisms in $\mathbb{A}, \beta_{X}: \gamma_{X} \rightarrow \gamma_{X}^{\prime}$, indexed in the objects $X$ of $\mathbb{G}$ ) such that

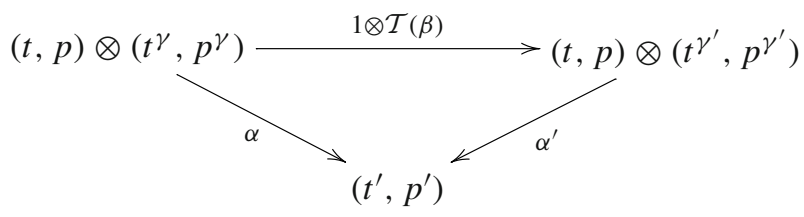

commutes, where, for any morphism $f: X \otimes Y \rightarrow Z$ in $\mathbb{G}, \mathcal{T}(\beta)_{f}: p^{\gamma}(f) \rightarrow p^{\gamma^{\prime}}(f)$ is given by $\mathcal{T}(\beta)_{f}={ }^{X} \beta_{Y} \otimes \beta_{X} \otimes \beta_{Z}^{*}$. Then the morphism $\overline{\mathcal{E}}(\beta): \overline{\mathcal{E}}(\gamma, \alpha) \rightarrow \overline{\mathcal{E}}\left(\gamma^{\prime}, \alpha^{\prime}\right)$ is described in the diagram

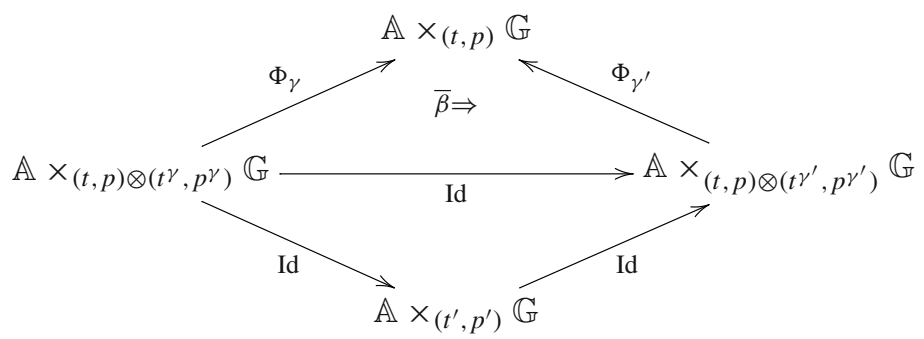


where $\bar{\beta}_{A, X}:\left(A \otimes \gamma_{X}, X\right) \rightarrow\left(A \otimes \gamma_{X}^{\prime}, X\right)$ is given, since $p\left(1_{X} l_{X}\right) \cong I$ and up to a canonical morphism, by the pair $\left(1_{X}, 1_{A} \otimes \beta_{X}\right)$. The monoidal structure on the identity functor Id is determined by $1 \otimes \mathcal{T}(\beta)$ as in Step 1 .

Finally, the fact that the identity natural transformation in the bottom triangle is monoidal is precisely condition (3). Also, the natural transformation $\bar{\beta}$ is monoidal and this fact follows from the observation that the morphism involved in such condition

$$
\Phi_{\gamma^{\prime}} I \mathrm{~d}((A, X) \otimes(B, Y)) \longrightarrow \Phi_{\gamma^{\prime}} I \mathrm{~d}((A, X)) \otimes \Phi_{\gamma^{\prime}} I \mathrm{~d}((B, Y))
$$

is determined, since $p^{\gamma}\left(1_{X \otimes Y}\right)={ }^{X} \gamma_{Y} \otimes \gamma_{X} \otimes \gamma_{X \otimes Y}^{*}$, by the following composition

$$
X_{\gamma_{Y}} \otimes \gamma_{X} \otimes \gamma_{X \otimes Y}^{*} \otimes \gamma_{X \otimes Y}^{\prime} \stackrel{1 \otimes \beta_{X \otimes Y}^{*} \otimes 1}{\longrightarrow} X_{\gamma_{Y}} \otimes \gamma_{X} \otimes \gamma_{X \otimes Y}^{\prime *} \otimes \gamma_{X \otimes Y}^{\prime} \stackrel{{ }^{X} \beta_{Y} \otimes \beta_{X} \otimes \mathrm{can}}{\longrightarrow} X_{\gamma_{Y}^{\prime}} \otimes \gamma_{X}^{\prime} .
$$

Step 4 Finally, we have to prove that the homomorphism $\overline{\mathcal{E}}: \mathcal{H}^{2}(\mathbb{G}, \mathbb{A}) \rightarrow$ $\mathbb{O} \operatorname{pext}(\varphi, \mathbb{G}, \mathbb{A})$, constructed in Step 3 , is a biequivalence, that is, it is locally an equivalence and biessentially surjective on objects (i.e., surjective up to equivalence). $\overline{\mathcal{E}}$ is locally faithful:

If $\beta, \beta^{\prime}:(\gamma, \alpha) \rightarrow\left(\gamma^{\prime}, \alpha^{\prime}\right)$ are such that $\overline{\mathcal{E}}(\beta)=\overline{\mathcal{E}}\left(\beta^{\prime}\right)$, then for all $A \in \mathbb{A}$ and $X \in \mathbb{G}$ we have

$$
1_{A} \otimes \beta_{X}=1_{A} \otimes \beta_{X}^{\prime}: A \otimes \gamma_{X} \rightarrow A \otimes \gamma_{X}^{\prime}
$$

and taking $A=I$ we get $\beta_{X}=\beta_{X}^{\prime}$ and so $\beta=\beta^{\prime}$.

$\overline{\mathcal{E}}$ is locally full:

Suppose $(\gamma, \alpha),\left(\gamma^{\prime}, \alpha^{\prime}\right):(t, p) \longrightarrow\left(t^{\prime}, p^{\prime}\right)$ two 1-arrows in $\mathcal{H}^{2}(\mathbb{G}, \mathbb{A})$ and let us suppose that $\bar{\beta}: \overline{\mathcal{E}}(\gamma, \alpha) \Rightarrow \overline{\mathcal{E}}\left(\gamma^{\prime}, \alpha^{\prime}\right)$ is a 2-arrow between both morphisms of singular extensions from $\mathcal{E}(t, p)$ to $\mathcal{E}\left(t^{\prime}, p^{\prime}\right)$. We look for a 2-arrow in $\mathcal{H}^{2}(\mathbb{G}, \mathbb{A})$, $\beta:(\gamma, \alpha) \longrightarrow\left(\gamma^{\prime}, \alpha^{\prime}\right)$ such that $\overline{\mathcal{E}}(\beta)=\bar{\beta}$. Explicitly, $\bar{\beta}$ is a natural transformation

$$
\bar{\beta}_{A, X}: \Phi_{\gamma}(A, X)=\left(A \otimes \gamma_{X}, X\right) \longrightarrow\left(A \otimes \gamma_{X}^{\prime}, X\right)=\Phi_{\gamma^{\prime}}(A, X)
$$

and so it has two components

$$
\bar{\beta}_{1}(A, X): A \otimes \gamma_{X} \longrightarrow A \otimes \gamma_{X}^{\prime}, \quad \bar{\beta}_{2}(A, X): X \rightarrow X
$$

We define $\beta_{X}: \gamma_{X} \longrightarrow \gamma_{X}^{\prime}$ as follows

$$
\gamma_{X} \cong I \otimes \gamma_{X} \stackrel{\bar{\beta}_{1}(I, X)}{\longrightarrow} I \otimes \gamma_{X}^{\prime} \cong \gamma_{X}^{\prime}
$$

In this way, condition (pcm5) in [13] on $\bar{\beta}$ gives the normalization condition for $\beta: \gamma \rightarrow \gamma^{\prime}$ being a morphism in $\mathcal{C}^{1}(\mathbb{G}, \mathbb{A})$ and the monoidal character of $\bar{\beta}$ gives condition (3) so that $\beta:(\gamma, \alpha) \longrightarrow\left(\gamma^{\prime}, \alpha^{\prime}\right)$ is a 2-arrow in $\mathcal{H}^{2}(\mathbb{G}, \mathbb{A})$. Moreover, condition (act10) in [13] on $\bar{\beta}$ gives that $\bar{\beta}_{1}(A, X)=1 \otimes \beta_{X}: A \otimes \gamma_{X} \longrightarrow A \otimes \gamma_{X}^{\prime}$ 
and the fact that $\bar{\beta}$ is a morphism of extensions gives that $\bar{\beta}_{2}(A, X)=1_{X}: X \rightarrow X$. Thus, by definition, $\overline{\mathcal{E}}(\beta)=\bar{\beta}$.

$\overline{\mathcal{E}}$ is locally essentially surjective:

Consider two cocycles $(t, p),\left(t^{\prime}, p^{\prime}\right) \in \mathcal{Z}^{2}(\mathbb{G}, \mathbb{A})$ and $(R, \tau, \mu): \overline{\mathcal{E}}\left(t^{\prime}, p^{\prime}\right) \rightarrow$ $\overline{\mathcal{E}}(t, p)$ a homomorphism in $\mathbb{O} \operatorname{pext}(\varphi, \mathbb{G}, \mathbb{A})$

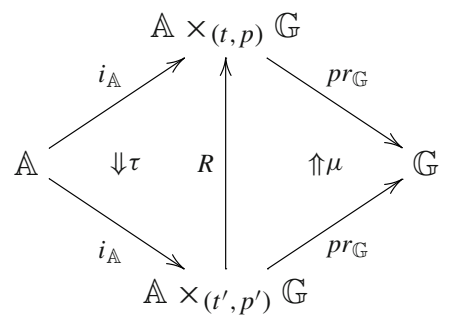

We are going to construct an 1 -arrow $(\gamma, \alpha)$ in $\mathcal{H}^{2}(\mathbb{G}, \mathbb{A})$, that is, $\gamma \in \mathcal{C}^{1}(\mathbb{G}, \mathbb{A})$ and $\alpha:(t, p) \otimes\left(t^{\gamma}, p^{\gamma}\right) \rightarrow\left(t^{\prime}, p^{\prime}\right)$ an arrow in $\mathbb{Z}^{2}(\mathbb{G}, \mathbb{A})$, and $\beta: \Phi_{\gamma} \Rightarrow R$ a morphism in $\mathbb{O} \operatorname{pext}(\varphi, \mathbb{G}, \mathbb{A})$. Let us write

$$
R(A, X)=\left(R^{1}(A, X), R^{2}(A, X)\right)
$$

for the two components of $R$, and

$$
\tau_{A}=\left(\tau^{1}(A), \tau^{2}(A)\right):(A, I) \rightarrow\left(R^{1}(A, I), R^{2}(A, I)\right)
$$

for the two components of $\tau$, and observe that

$$
\mu_{A, I}=\tau^{2}(A): I \rightarrow R^{2}(A, I)
$$

because of condition (ext2) in [13], and

$$
\tau^{1}(A): A \otimes p\left(\mu_{A, I} l\right) \longrightarrow R^{1}(A, I)
$$

Now we put

$$
\gamma: \operatorname{Obj}(\mathbb{G}) \rightarrow \operatorname{Obj}(\mathbb{A}) \quad \text { by } \quad \gamma_{X}=R^{1}(I, X) \otimes p\left(\mu_{I, X} l\right)^{*} \in \operatorname{Obj}(\mathbb{A})
$$

As far as the natural transformation $\beta$ is concerned, it is of the form

$\beta_{A, X}=\left(\beta^{1}(A, X), \beta^{2}(A, X)\right): \Phi_{\gamma}(A, X)=\left(A \otimes \gamma_{X}, X\right) \rightarrow\left(R^{1}(A, X), R^{2}(A, X)\right)$

and we put

$$
\beta^{2}(A, X)=\mu_{A, X}: X \rightarrow R^{2}(A, X)
$$


To construct the first component $\beta^{1}(A, X): A \otimes \gamma_{X} \otimes p\left(\mu_{A, X} l\right) \longrightarrow R^{1}(A, X)$, observe that for any cocycle $(t, p)$ one has $(A, I) \otimes_{(t, p)}(I, X) \cong\left(A \otimes p\left(1_{I \otimes X}\right), I \otimes\right.$ $X) \cong(A, X)$ where the second isomorphism is given by the morphism $t: p(l l) \otimes$ $p\left(1_{I \otimes X}\right) \longrightarrow I$ determined by the following commutative diagram

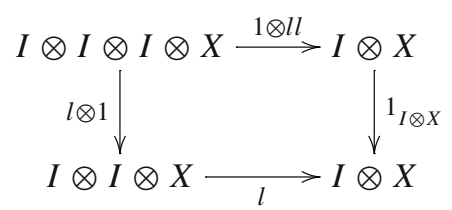

Thus the monoidal structure of $R$ provides a natural family of arrows $R_{2}^{A, X}$ with two components of the form

$$
\begin{aligned}
& \rho^{2}(A, X): R^{2}(A, I) \otimes R^{2}(I, X) \longrightarrow R^{2}(A, X), \\
& \rho^{1}(A, X): R^{1}(A, I) \otimes R^{1}(I, X) \otimes p\left(1_{R^{2}(A, I) \otimes R^{2}(I, X)}\right) \\
& \quad \otimes p\left(\rho^{2}(A, X) l\right) \longrightarrow R^{1}(A, X)
\end{aligned}
$$

and we put $\beta^{1}(A, X)$ as the following composite

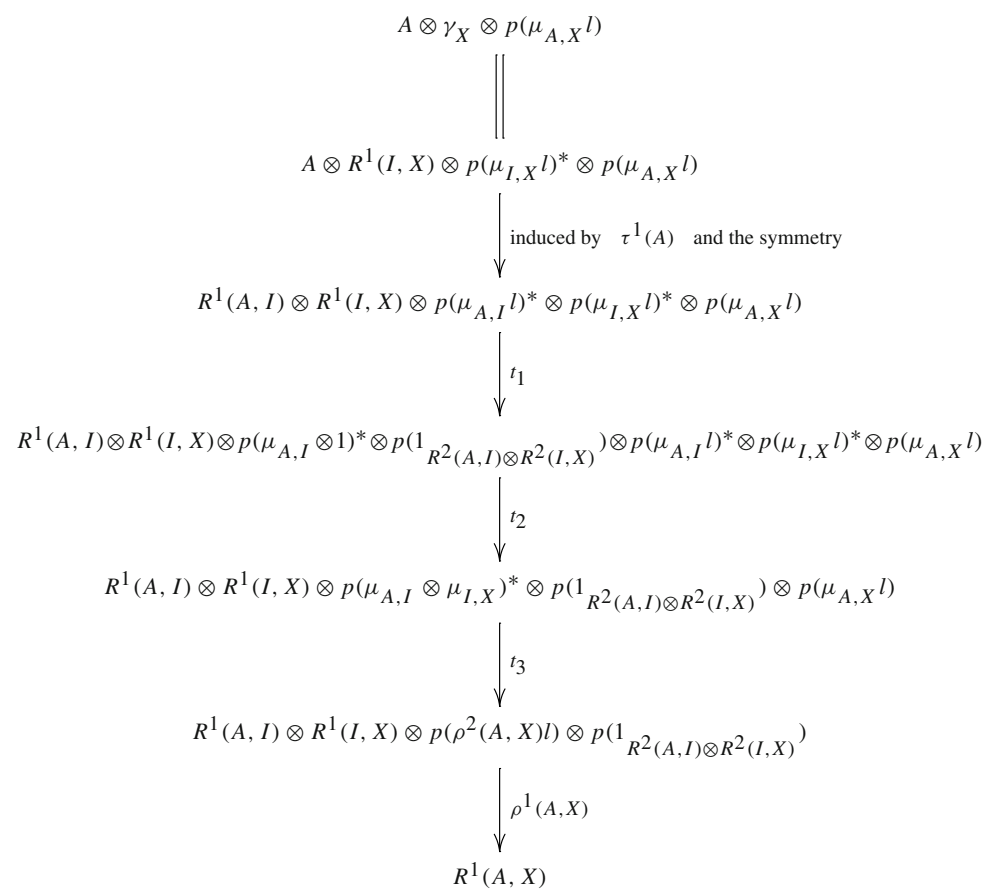


where $t_{i}, i=1,2,3$, are respectively determined by the following commutative diagrams:
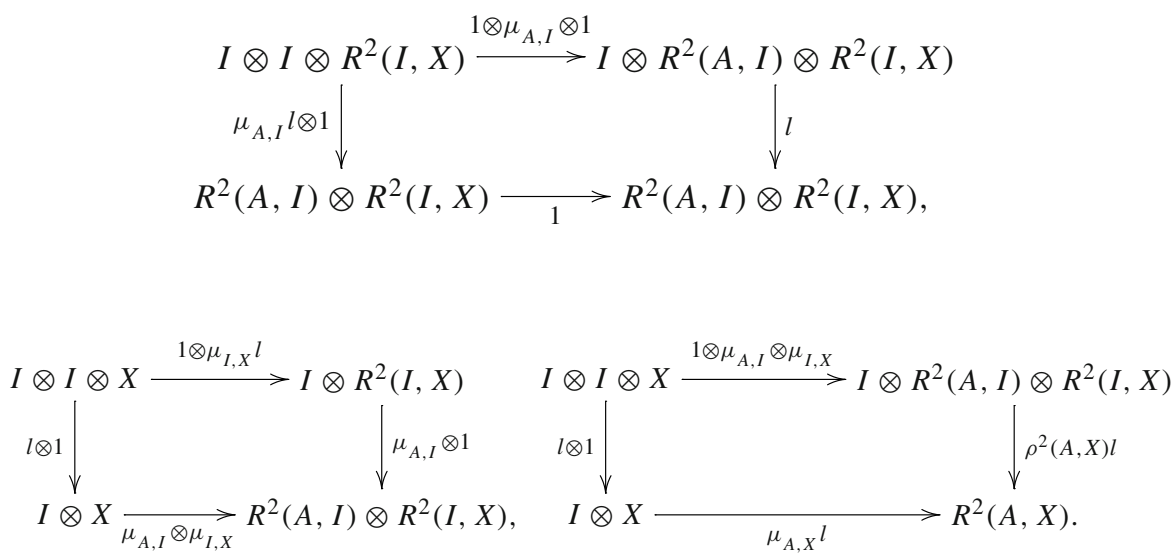

Finally, to construct the arrow $\alpha:(t, p) \otimes\left(t^{\gamma}, p^{\gamma}\right) \rightarrow\left(t^{\prime}, p^{\prime}\right)$ in $\mathbb{Z}^{2}(\mathbb{G}, \mathbb{A})$ we need to give, for any morphism in $\mathbb{G}, f: X \otimes Y \longrightarrow Z$, a morphism in $\mathbb{A}$

$$
\alpha_{f}: p(f) \otimes p^{\gamma}(f) \longrightarrow p^{\prime}(f)
$$

which we give as the following composite:

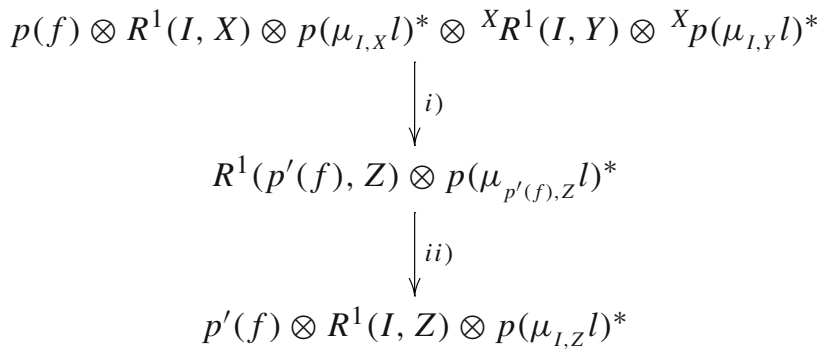

where:

- the morphism ii) is induced by

$$
\beta^{1}\left(p^{\prime}(f), Z\right)^{-1}: R^{1}\left(p^{\prime}(f), Z\right) \longrightarrow p^{\prime}(f) \otimes R^{1}(I, Z) \otimes p\left(\mu_{I, Z} l\right)^{*} \otimes p\left(\mu_{p^{\prime}(f), Z} l\right)
$$


- The morphism i) is given as the following composite (as usual, symmetry in $\mathbb{A}$ is freely used):

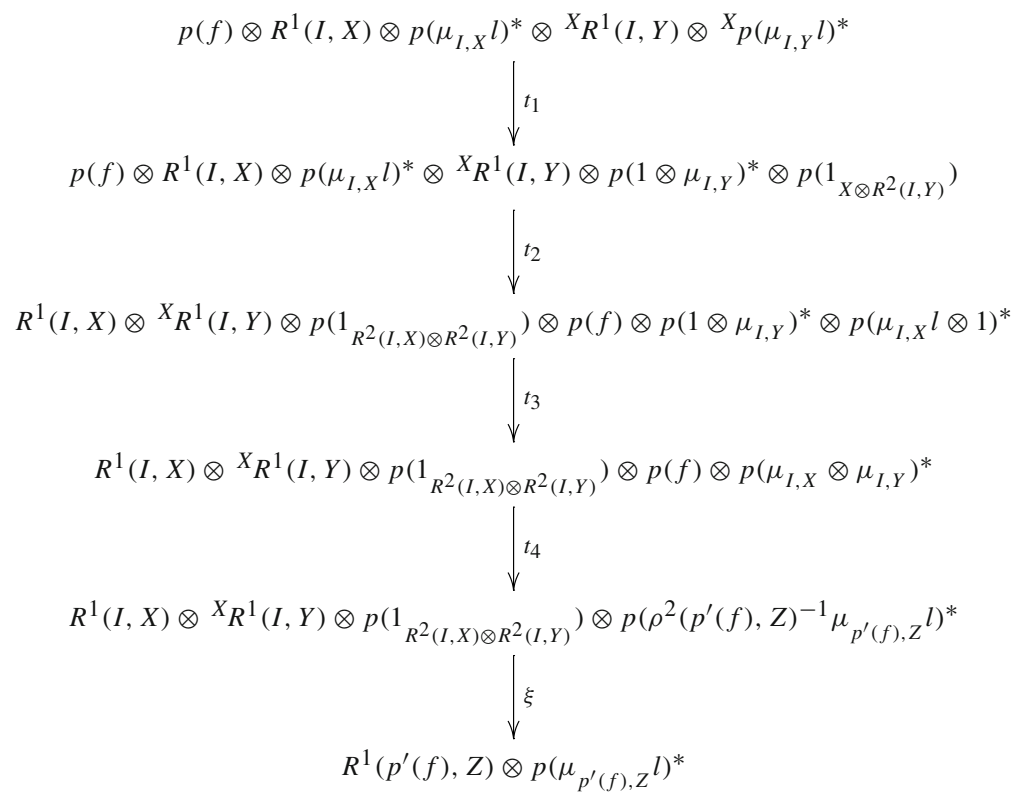

where $t_{i}, i=1,2,3,4$, are respectively determined by the following commutative diagrams
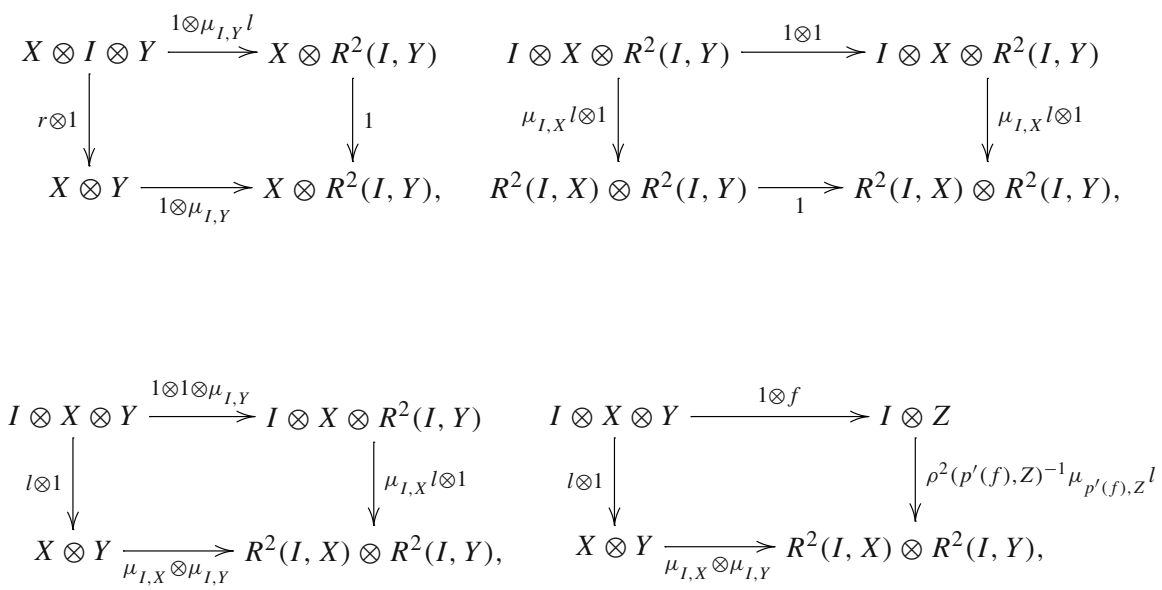

and $\xi$ is deduced from the following arrow, 


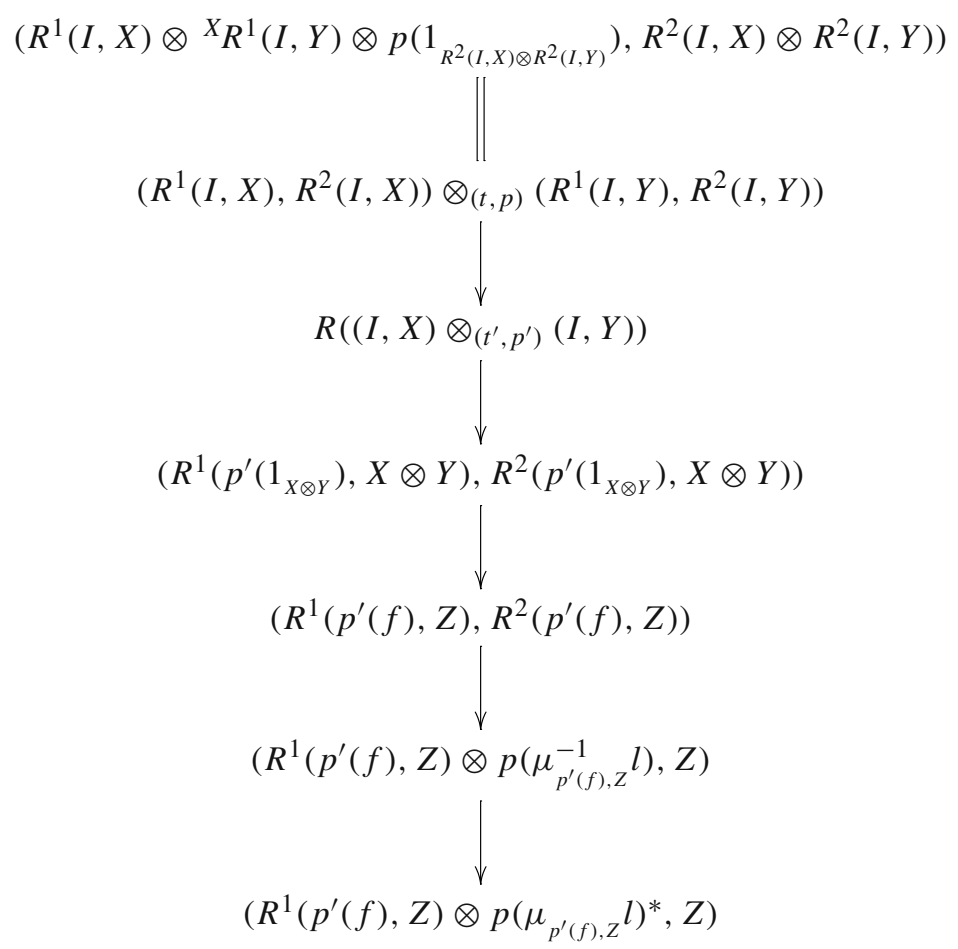

where we use the definition of $\otimes_{(t, p)}$ in the first step, the monoidal structure of $R$ in the second step, $\varphi_{\bullet}^{X}$ and the definition of $\otimes_{\left(t^{\prime}, p^{\prime}\right)}$ in the third step, the existence of the morphism $(I, X) \otimes_{\left(t^{\prime}, p^{\prime}\right)}(I, Y) \longrightarrow\left(p^{\prime}(f), Z\right)$ associated with the morphism in $\mathbb{A}$, $p^{\prime}\left(1_{X \otimes Y}\right) \otimes p^{\prime}(f l) \longrightarrow p^{\prime}(f)$, deduced from the commutative diagram

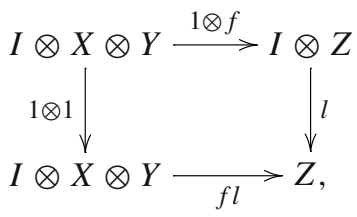

in the fourth step, the morphism in $\mathbb{A} \times{ }_{\left(t^{\prime}, p^{\prime}\right)} \mathbb{G}$

$\left(1, \mu_{p^{\prime}(f), Z}^{-1}\right):\left(R^{1}\left(p^{\prime}(f), Z\right), R^{2}\left(p^{\prime}(f), Z\right)\right) \longrightarrow\left(R^{1}\left(p^{\prime}(f), Z\right) \otimes p\left(\mu_{p^{\prime}(f), Z}^{-1} l\right), Z\right)$

in the fifth step and, in the last step, the existence of the morphism in $\mathbb{A}$,

$$
p\left(\mu_{p^{\prime}(f), Z}^{-1} l\right) \longrightarrow p\left(\mu_{p^{\prime}(f), Z} l\right)^{*}
$$


determined by the commutative diagram

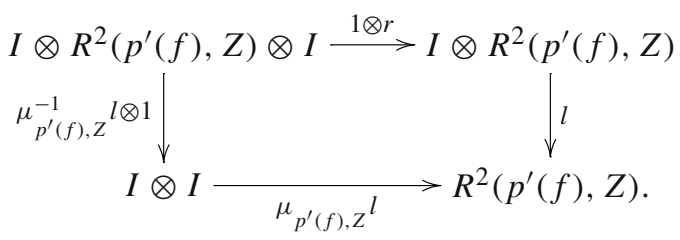

$\overline{\mathcal{E}}$ is biessentially surjective:

We have to prove that $\overline{\mathcal{E}}$ is surjective up to equivalence. Let

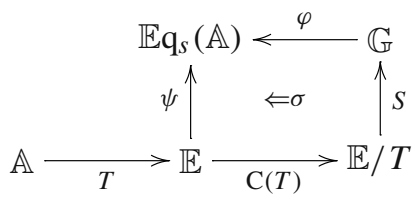

be a $\varphi$-extension. Up to the equivalences $\mathbb{G} \simeq \mathbb{E} / T$ we can describe a cocycle $(t, p) \in$ $\left.\mathcal{Z}^{2}(\mathbb{E} / T, \mathbb{A})\right)$ as follows. Any morphism $[A, f]: X \otimes Y \longrightarrow Z$ in $\mathbb{E} / T$ is an equivalence class of prearrows $(A \in \mathbb{A}, X \otimes Y \stackrel{f}{\longrightarrow} T(A) \otimes Z)$ and we choose $p([A, f])=$ $A$ (and we denote $A=p(f)$ ). Also, recalling that the identity in an object $X$ is the class $[I$, can $: X \rightarrow T(I) \otimes X]$ we have that, for any commutative diagram in $\mathbb{E} / T$ as

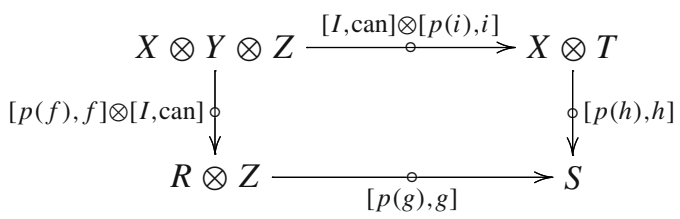

and, according to the definition of the tensor product of morphisms in the quotient categorical group (see Sect. 2), the object part of the composite $[p(h), h] \cdot([I, c a n] \otimes$ $[p(i), i])$ is, unless canonical isomorphisms, ${ }^{X} p(i) \otimes p(h)$, whereas the object part of the composite $[p(g), g] \cdot([p(f), f] \otimes[I, c a n])$ is, again unless canonical isomorphisms, $p(f) \otimes p(g)$. Then the commutativity of the square implies the existence of a morphism in $\mathbb{A}$

$$
t_{[p(f), f],[p(g), g],[p(h), h],[p(i), i]}:{ }^{X} p(i) \otimes p(h) \longrightarrow p(f) \otimes p(g)
$$

such that the following diagram in $\mathbb{E}$ is commutative

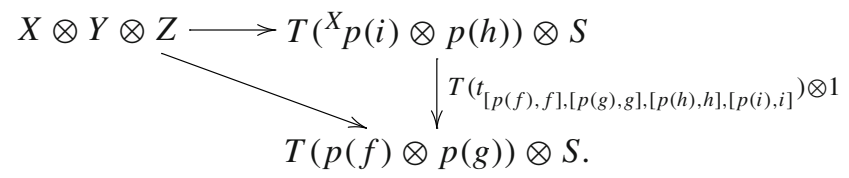


In this way it is straightforward to check that the pair $(t, p) \in \mathcal{Z}^{2}(\mathbb{G}, \mathbb{A})$. Moreover, any other choice of the objects $p(f)$ gives a cohomologous 2-cocycle, that is, isomorphic in the quotient $\mathcal{H}^{2}(\mathbb{G}, \mathbb{A})$. We finish by showing that $\overline{\mathcal{E}}(t, p)$ is isomorphic to the given $\varphi$-extension. The needed arrow in $\mathbb{O} \operatorname{pext}(\varphi, \mathbb{G}, \mathbb{A})$ is given by

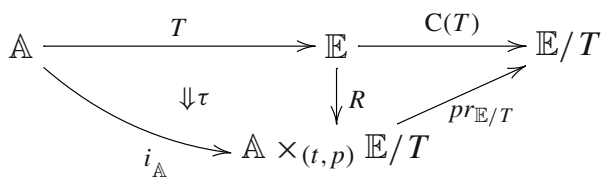

with $R(X)=(I, X)$ and $\tau_{A}:(I, T(A)) \longrightarrow(A, I)$ the morphism in $\mathbb{A} \times_{(t, p)} \mathbb{E} / T$ determined by the arrow in $\mathbb{E} / T\left[A, r_{T(A)}^{-1}\right]: T(A) \longrightarrow \rightarrow$ and the arrow in $\mathbb{A}$ $I \otimes p\left(r_{T(A)}^{-1} l\right)=I \otimes A \stackrel{c a n}{\longrightarrow} A$.

The Baer sum of singular extensions of a group $G$ by a $G$-module $A$ can be defined in a direct way or, alternatively in an indirect way, by using the bijection of the set of classes of such extensions with the second cohomology group $H^{2}(G, A)$. This second approach can be used to define also a Baer sum in the categorical group case. The biequivalence $\bar{\epsilon}$ of previous theorem induces an equivalence between the respective groupoids obtained by taking 2 -isomorphisms classes of 1-arrows as arrows. Each pseudo-inverse of this equivalence certainly allows to define, using the tensor product in the symmetric categorical group $\mathcal{H}^{2}(\mathbb{G}, \mathbb{A})$, a symmetric categorical group structure (i.e., a Baer sum) on the groupoid $\operatorname{Opext}(\varphi, \mathbb{G}, \mathbb{A})$ and, of course, all these different structures yield to equivalent symmetric categorigal groups. In summary we get the following corollary.

Corollary 3.6 Let $\mathbb{G}$ be a categorical group and $\varphi: \mathbb{G} \rightarrow \mathbb{E} \mathrm{q}_{s}(\mathbb{A})$ a symmetric action. Each weak-inverse of the biequivalence

$$
\overline{\mathcal{E}}: \mathcal{H}^{2}(\mathbb{G}, \mathbb{A}) \rightarrow \mathbb{O} \operatorname{pext}(\varphi, \mathbb{G}, \mathbb{A})
$$

induces a structure of symmetric categorical group on the groupoid $\operatorname{Opext}(\varphi, \mathbb{G}, \mathbb{A})$ an all of them are equivalent.

Remark 3.7 With the appropriate notion of morphism between those singular extensions recalled in Remark 3.2 (introduced in [9, Definition 4.1]), the category of singular extensions $\mathcal{E}(\mathbb{G}, \mathbb{A})$ was also considered in [9] where, actually, it was proved the existence of a bijection $\left[9\right.$, Theorem 4.4] $\pi_{0}(\mathcal{E}(\mathbb{G}, \mathbb{A})) \cong \mathbf{H}^{1}(\mathbb{G}, \mathbb{A})$ of its connected components with the first cohomology group there introduced. Comparing the construction of this $\mathbf{H}^{1}$ with our definition of the categorical group $\mathcal{H}^{2}(\mathbb{G}, \mathbb{A})$, we see that $\mathbf{H}^{1}(\mathbb{G}, \mathbb{A}) \simeq \pi_{0}\left(\mathcal{H}^{2}(\mathbb{G}, \mathbb{A})\right)$ and then this isomorphism provides, at this group-level, an alternative and dual point of view of the classification theorem 3.5. In that way, singular extensions in terms of surjective fibrations and fibre categories or, equivalently (c.f. [20]), of essentially surjective homomorphisms and their homotopy kernels, would be considered instead of those singular extensions studied in this paper which are defined in terms of faithful homomorphisms and their homotopy cokernels. 


\section{A new Hochschild-Serre 2-exact sequence}

In Corollary 6.5 of [13] we showed categorical group versions of the classical Hochschild-Serre 5-term exact group sequences [14]. These categorical versions involve derivations and a second categorical cohomology group $\mathbb{H}^{2}$ able of classifying the singular extensions of categorical groups with symmetric kernel and a functorial section. In order to classify all singular extensions, we have introduced in Sect. 3 a new second categorical cohomology group $\mathcal{H}^{2}$ and now, in this section, our aim is to show that, involving this $\mathcal{H}^{2}$, there are also corresponding categorical group versions of the classical 5-term Hochschild-Serre sequences.

We fix an essentially surjective homomorphism of categorical groups $P: \mathbb{E} \rightarrow \mathbb{G}$, a symmetric $\mathbb{G}$-categorical group $\mathbb{A}=\left(\mathbb{A}, \varphi: \mathbb{G} \rightarrow \mathbb{E} \mathrm{q}_{s}(\mathbb{A})\right)$ and we consider the faithful homomorphisms given by composition with $P, P_{\mathbb{C}}: \mathcal{C}^{1}(\mathbb{G}, \mathbb{A}) \rightarrow \mathcal{C}^{1}(\mathbb{E}, \mathbb{A})$ and $P_{\mathbb{Z}}: \mathcal{Z}^{2}(\mathbb{G}, \mathbb{A}) \rightarrow \mathcal{Z}^{2}(\mathbb{E}, \mathbb{A})$.

Note that the kernel of the homomorphism $\mathcal{T}: \mathcal{C}^{1}(\mathbb{G}, \mathbb{A}) \rightarrow \mathcal{Z}^{2}(\mathbb{G}, \mathbb{A})$ is the symmetric categorical group $\mathcal{D}(\mathbb{G}, \mathbb{A})$ that we call of the 'non-functorial' derivations. This name is justified because its objects are maps $D: \operatorname{Obj}(\mathbb{G}) \rightarrow \operatorname{Obj}(\mathbb{A})$ together with a family of isomorphisms

$$
\left\{\beta=\beta_{f}: D(X) \otimes{ }^{X} D(Y) \rightarrow D(Z)\right\}_{\{f: X \otimes Y \rightarrow Z \in \operatorname{Mor}(\mathbb{G})\}}
$$

such that, for any commutative diagram as (1), the following diagram is commutative:

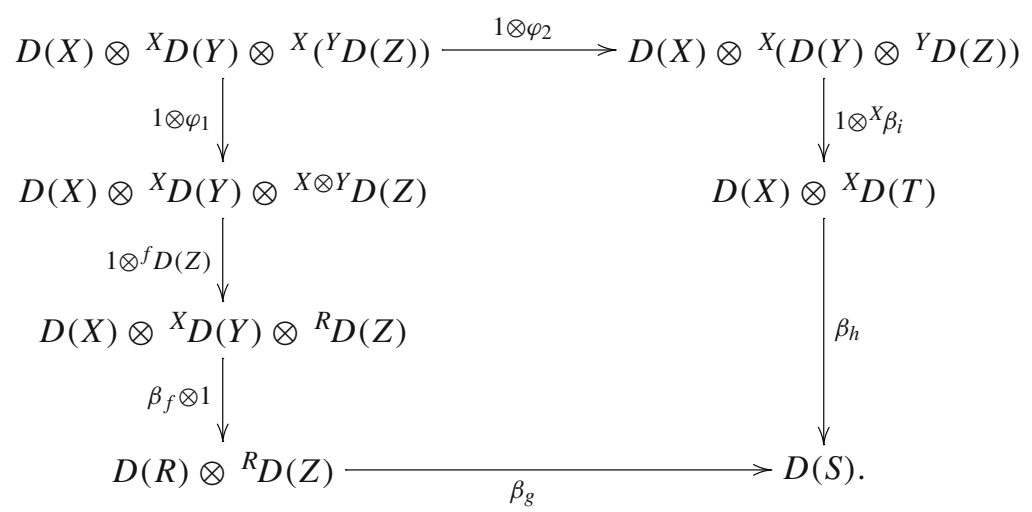

An arrow in $\mathcal{D}(\mathbb{G}, \mathbb{A})$ from $(D, \beta)$ to $\left(D^{\prime}, \beta^{\prime}\right)$ is a family of isomorphisms

$$
\left\{\epsilon_{X}: D(X) \longrightarrow D^{\prime}(X)\right\}_{X \in \mathbb{G}}
$$

such that, for any morphism $f: X \otimes Y \rightarrow Z$ in $\mathbb{G}$, the following condition holds:

$$
\beta_{f}^{\prime} \cdot\left(\epsilon_{X} \otimes{ }^{X} \epsilon_{Y}\right)=\epsilon_{Z} \cdot \beta_{f} .
$$

Of course, the symmetric categorical group structure in $\mathcal{D}(\mathbb{G}, \mathbb{A})$ is induced by that of $\mathbb{A}$. 
Remark 4.1 When the action of $\mathbb{G}$ on $\mathbb{A}$ is the trivial one, the symmetric categorical groups $\mathcal{D}(\mathbb{G}, \mathbb{A})$ of 'non-functorial derivations' becomes a categorical group of 'nonfunctorial homomorphisms' from $\mathbb{G}$ to $\mathbb{A}$ that we will denote by $n f \mathcal{H o m}(\mathbb{G}, \mathbb{A})$. Its objects are pairs $\left(F, F_{2}\right)$ where $F$ is a map $F: \operatorname{Obj}(\mathbb{G}) \rightarrow \operatorname{Obj}(\mathbb{A})$ and

$$
F_{2}=F_{2}^{f}: F(X) \otimes F(Y) \rightarrow F(Z)
$$

is a family of isomorphisms indexed in all morphisms $f: X \otimes Y \rightarrow Z$ in $\mathbb{G}$, such that, for any commutative diagram as (1), the following condition holds

$$
F_{2}^{h} \cdot\left(1 \otimes F_{2}^{i}\right)=F_{2}^{g} \cdot\left(F_{2}^{f} \otimes 1\right)
$$

There exists then a morphism $F_{0}: I \rightarrow F(I)$ such that, for any $X \in \mathbb{G}$, the equalities $F_{2}^{r} \cdot\left(1 \otimes F_{0}\right)=r$ and $F_{2}^{l} \cdot\left(F_{0} \otimes 1\right)=l$ hold.

In particular, there always exists a morphism $F_{2}^{1_{X \otimes Y}}: F_{2}(X) \otimes F_{2}(Y) \rightarrow F_{2}(X \otimes Y)$ and therefore, for any morphism $f: X \otimes Y \rightarrow Z$ in $\mathbb{G}$ and considering the induced one $\widetilde{f}: X \otimes Y \otimes Z^{*} \rightarrow I$, there exists also a morphism $F\left(X \otimes Y \otimes Z^{*}\right) \rightarrow I$ canonically deduced from the one $F_{2}^{l \cdot(\widetilde{f} \otimes 1)}: F\left(X \otimes Y \otimes Z^{*}\right) \otimes F(I) \rightarrow F(I)$.

A morphism in $n f \mathcal{H} \operatorname{om}(\mathbb{G}, \mathbb{A})$ from $\left(F, F_{2}\right)$ to $\left(F^{\prime}, F_{2}^{\prime}\right)$ is a family of isomorphisms $\left\{\tau_{X}: F(X) \longrightarrow F^{\prime}(X)\right\}_{X \in \mathbb{G}}$ such that, for any morphism $f: X \otimes Y \rightarrow Z$ in $\mathbb{G}$, the following condition holds

$$
F_{2}^{\prime f} \cdot\left(\tau_{X} \otimes{ }^{X} \tau_{Y}\right)=\tau_{Z} \cdot F_{2}^{f}
$$

Considering the kernel and cokernel of the homomorphism $\mathcal{T}: \mathcal{C}^{1}(\mathbb{G}, \mathbb{A}) \rightarrow$ $\mathcal{Z}^{2}(\mathbb{G}, \mathbb{A})$, we have the following 2-exact sequence

$$
\mathcal{D}(\mathbb{G}, \mathbb{A}) \stackrel{K(\mathcal{T})}{\longrightarrow} \mathcal{C}^{1}(\mathbb{G}, \mathbb{A}) \stackrel{\mathcal{T}}{\longrightarrow} \mathcal{Z}^{2}(\mathbb{G}, \mathbb{A}) \stackrel{C(\mathcal{T})}{\longrightarrow} \mathcal{H}^{2}(\mathbb{G}, \mathbb{A}) .
$$

There is also a homomorphism of symmetric categorical groups, $d_{\mathbb{G}}: \mathbb{A} \longrightarrow$ $\mathcal{D}(\mathbb{G}, \mathbb{A})$, given by inner derivations, that is, for any $A \in \mathbb{A}$ and $X \in \mathbb{G}, d_{\mathbb{G}}(A)(X)=$ ${ }^{X} A \otimes A^{*}$.

The cokernel $\operatorname{Coker}\left(d_{\mathbb{G}}\right)$ deserves to be called the first cohomology categorical group of $\mathbb{G}$ with coefficients in $\mathbb{A}$ and will be denoted by $\mathcal{H}^{1}(\mathbb{G}, \mathbb{A})$.

Now, following the same steps as in Section 5 of [13], we can construct the following diagram in $\mathcal{S C G}$ : 


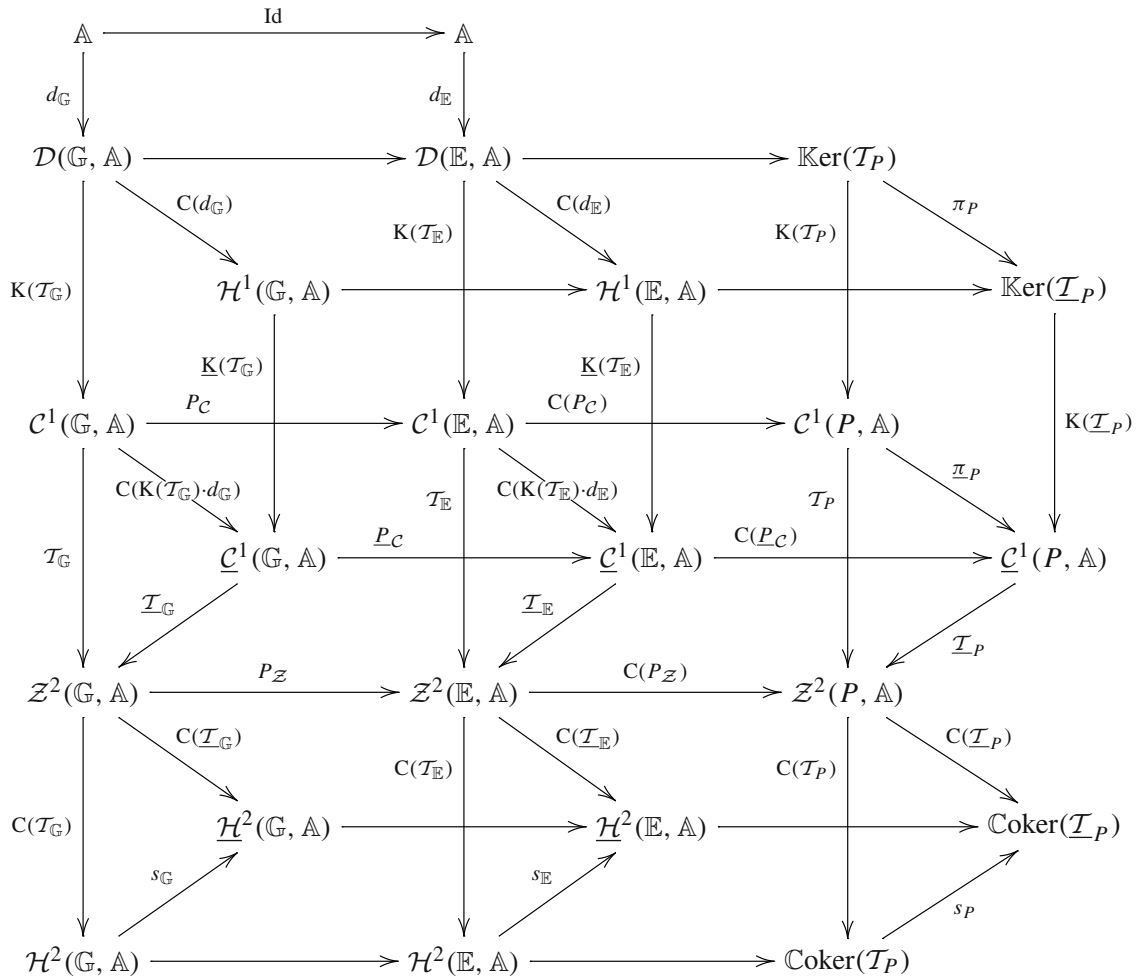

where

$-\underline{\mathcal{C}}^{1}(\mathbb{G}, \mathbb{A})$ is the cokernel of $\mathrm{K}\left(\mathcal{T}_{\mathbb{G}}\right) \cdot d_{\mathbb{G}}$ and $\underline{\mathcal{H}}^{2}(\mathbb{G}, \mathbb{A})$ is the cokernel of $\underline{\mathcal{T}} \underset{\mathbb{G}}{ }$;

$-\overline{\mathcal{C}}^{1}(P, \mathbb{A})$ is the cokernel of $P_{\mathcal{C}}, \underline{\mathcal{C}}^{1}(P, \mathbb{A})$ is the cokernel of $\underline{P}_{\mathcal{C}}$, and $\mathcal{Z}^{2}(P, \mathbb{A})$ is the cokernel of $P_{\mathcal{Z}}$.

Now, the proof of the next proposition is identical to that of Proposition 5.4 in [13].

Proposition 4.2 Let $P: \mathbb{E} \rightarrow \mathbb{G}$ be an essentially surjective homomorphism of categorical groups and $\mathbb{A}$ a symmetric $\mathbb{G}$-categorical group. There exists a diagram of 2-exact sequences of symmetric categorical groups

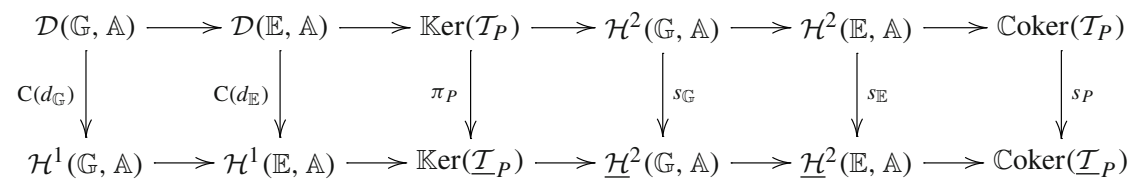

where $\mathrm{C}\left(d_{\mathbb{G}}\right)$ and $\mathrm{C}\left(d_{\mathbb{E}}\right)$ are essentially surjective, $s_{\mathbb{G}}$ and $s_{\mathbb{E}}$ are full and essentially surjective and $\pi_{P}$ and $s_{P}$ are equivalences.

We give now a suitable interpretation of the third point of these sequences (c.f. Proposition 6.4 in [13]) in order to get the desired new 2-dimensional HochschildSerre sequences involving the second cohomology categorical group $\mathcal{H}^{2}$. For this, let 
$\mathbb{K}$ be the kernel of the fixed homomorphism $P: \mathbb{E} \rightarrow \mathbb{G}$ and recall, from Example 2.6.v in [10], that there is a canonical action $\bullet: \mathbb{E} \times \mathbb{K} \rightarrow \mathbb{K}$ with constraints $\bullet_{0}, \bullet_{1}, \bullet_{2}$. Explicitly,

$$
E \bullet\left(K, u_{K}: P K \rightarrow I\right)=\left(E \otimes K \otimes E^{*}, u_{E \otimes K \otimes E^{*}}\right)
$$

where

$$
u_{E \otimes K \otimes E^{*}}=P\left(E \otimes K \otimes E^{*}\right) \stackrel{P_{2}^{-1}}{\longrightarrow} P E \otimes P K \otimes P E^{*} \stackrel{\mathrm{id} \otimes u_{K} \otimes \mathrm{id}}{\longrightarrow} P E \otimes I \otimes P E^{*} \simeq I .
$$

Let us consider now the symmetric categorical group

$$
\underline{\operatorname{nf} \mathcal{H o m}}(\mathbb{K}, \mathbb{A})
$$

having equivariant non-functorial homomorphisms from $\mathbb{K}$ to $\mathbb{A}$ as objects. This means that an object is a triplet $\left(F, F_{2}, F_{\varphi}\right)$ where $\left(F, F_{2}\right) \in n f \mathcal{H}$ om $(\mathbb{K}, \mathbb{A})$ (see Remark 4.1) and $F_{\varphi}$ is a family of arrows

$$
F_{\varphi}^{E,\left(K, u_{K}\right)}: F\left(E \bullet\left(K, u_{K}\right)\right) \rightarrow{ }^{P E} F\left(K, u_{K}\right)
$$

satisfying the following coherence conditions:

$$
\begin{aligned}
\text { (nf1) } & F_{\varphi}^{I,\left(K, u_{K}\right)} \cdot F_{2}\left(\bullet_{0}^{\left(K, u_{K}\right)} \cdot l\right) \cdot\left(\left(F_{0} \otimes 1\right) \cdot l^{-1}\right)=P_{0} F\left(K, u_{K}\right) \cdot \varphi_{0}^{F\left(K, u_{K}\right)} \\
\text { (nf2) } \quad & P E F_{2}^{i d_{\left(K, u_{K}\right) \otimes\left(J, u_{J}\right)}} \cdot \varphi_{2}^{P E, F\left(K, u_{K}\right), F\left(J, u_{J}\right)} \cdot\left(F_{\varphi}^{E,\left(K, u_{K}\right)} \otimes F_{\varphi}^{E,\left(J, u_{J}\right)}\right) \\
& =F_{\varphi}^{E,\left(K, u_{K}\right) \otimes\left(J, u_{J}\right)} \cdot F_{2}^{\bullet^{E,\left(K, u_{K}\right),\left(J, u_{J}\right)}} \\
(\text { nf3) } & P_{2}^{E, F} F\left(K, u_{K}\right) \cdot \varphi_{1}^{P E, P F, F\left(K, u_{K}\right)} \cdot P{ }^{E} F_{\varphi}^{F,\left(K, u_{K}\right)} \cdot F_{\varphi}^{E, F \bullet\left(K, u_{K}\right)}=F_{\varphi}^{E \otimes F,\left(K, u_{K}\right)} . \\
& F\left(\bullet_{1}^{E, F,\left(K, u_{K}\right)}\right)
\end{aligned}
$$

A morphism

$$
\tau:\left(F, F_{2}, F_{\varphi}\right) \Rightarrow\left(F^{\prime}, F_{2}^{\prime}, F_{\varphi}^{\prime}\right): \mathbb{K} \rightarrow \mathbb{A}
$$

in $n f \mathcal{H o m}(\mathbb{K}, \mathbb{A})$ is a morphism $\tau:\left(F, F_{2}\right) \rightarrow\left(F^{\prime}, F_{2}^{\prime}\right)$ in $n f \mathcal{H}$ om $(\mathbb{K}, \mathbb{A})$ such that (nf4) $P E_{\tau_{\left(K, u_{K}\right)}} \cdot F_{\varphi}^{E,\left(K, u_{K}\right)}=F_{\varphi}^{\prime E,\left(K, u_{K}\right)} \cdot \tau_{E \bullet\left(K, u_{K}\right)}$.

Next we see that this symmetric categorical group gives the desired interpretation of $\operatorname{Ker}\left(\mathcal{T}_{P}\right)$.

Theorem 4.3 Let $P: \mathbb{E} \rightarrow \mathbb{G}$ be an essentially surjective homomorphism of categorical groups with kernel $\mathbb{K}$ and let $\mathbb{A}$ be a symmetric $\mathbb{G}$-categorical group. Then there is an equivalence of symmetric categorical groups

$$
\underline{n f \mathcal{H o m}}(\mathbb{K}, \mathbb{A}) \simeq \mathbb{K} \operatorname{er}\left(\mathcal{T}_{P}\right)
$$


Proof Recall that an object of $\mathbb{K e r}\left(\mathcal{T}_{P}\right)$ consists of a pair $\left(\gamma, u_{\gamma}: \mathcal{T}_{P}(\gamma) \rightarrow 0\right)$ where $\gamma \in \operatorname{Obj}\left(\mathcal{C}^{1}(P, \mathbb{A})\right)=\operatorname{Obj}\left(\mathcal{C}^{1}(\mathbb{E}, \mathbb{A})\right)$, that is, $\gamma: \operatorname{Obj}(\mathbb{E}) \rightarrow \operatorname{Obj}(\mathbb{A}), X \mapsto \gamma_{X}$, is a map with $\gamma_{I} \cong I$, and $u_{\gamma}$ is a morphism in $\mathcal{Z}^{2}(P, \mathbb{A})$. This means that $u_{\gamma}$ is the class of a premorphism $\left((p, t), \mathcal{T}_{\mathbb{E}}(\gamma) \longrightarrow P_{\mathcal{Z}}(p, t) \otimes(0\right.$, can $\left.)\right)$ where $(p, t) \in \mathcal{Z}^{2}(\mathbb{G}, \mathbb{A})$ and $\mathcal{T}_{\mathbb{E}}(\gamma)=\left(p^{\gamma}, t^{\gamma}\right)$, with $p^{\gamma}(f)={ }^{P(X)} \gamma_{Y} \otimes \gamma_{X} \otimes \gamma_{Z}^{*}$ for any morphism $f: X \otimes Y \rightarrow Z$ in $\mathbb{E}$. Moreover, since $P$ is essentially surjective, we can choose, for any object $G \in \mathbb{G}$, an object $s G \in \mathbb{E}$ and an isomorphism $G \stackrel{e_{G}}{\longrightarrow} P(s G)$. In particular, we choose $s I=I$ with $e_{I}=P_{0}$ and, for any $G \in \mathbb{G}, s G^{*}=(s G)^{*}$ with $e_{G^{*}}=e_{G}^{*}$. Note that, for any object $X \in \mathbb{E}$, the object $X \otimes s P(X)^{*}$ together with the natural morphism $u_{X \otimes s(X)^{*}}: P\left(X \otimes s P(X)^{*}\right) \rightarrow P(X) \otimes P\left(s P(X)^{*}\right) \rightarrow P(X) \otimes P(X)^{*} \rightarrow I$ constitute an object of $\mathbb{K}$. Once this morphism is well-understood, in what follows we will omit it for simplicity.

Then, for any object $F=\left(F, F_{2}, F_{\varphi}\right) \in \underline{\text { nf } \mathcal{H} \text { om }}(\mathbb{K}, \mathbb{A})$, we define

$$
\Phi: \underline{\mathrm{nf}} \mathcal{H o m}(\mathbb{K}, \mathbb{A}) \longrightarrow \mathbb{K} \operatorname{er}\left(\mathcal{T}_{P}\right)
$$

by $\Phi(F)=\left(\gamma^{F}, u_{\gamma^{F}}\right)$ where, for any object $X$ in $\mathbb{E}, \gamma_{X}^{F}=F\left(X \otimes s P(X)^{*}\right.$. As for $u_{\gamma^{F}}$, it is given by the 2-cocycle $\left(p^{F}, t^{F}\right) \in \mathcal{Z}^{2}(\mathbb{G}, \mathbb{A})$ and the morphism in $\mathcal{Z}^{2}(\mathbb{E}, \mathbb{A})$, $\chi: \mathcal{T}_{\mathbb{E}}\left(\gamma^{F}\right) \longrightarrow P_{\mathcal{Z}}\left(p^{F}, t^{F}\right) \otimes(0$, can $)$, described below.

On the one hand, since for any morphism $g: G_{1} \otimes G_{2} \rightarrow G_{3}$ in $\mathbb{G}$ there is a morphism $u_{s G_{3} \otimes s G_{2}^{*} \otimes s G_{1}^{*}}: P\left(s G_{3} \otimes s G_{2}^{*} \otimes s G_{1}^{*}\right) \stackrel{e \cdot P_{2}}{\longrightarrow} G_{3} \otimes G_{2}^{*} \otimes G_{1}^{*} \stackrel{\widetilde{g}}{\longrightarrow} I$, the pair $\left(s G_{3} \otimes s G_{2}^{*} \otimes s G_{1}^{*}, u_{s G_{3} \otimes s G_{2}^{*} \otimes s G_{1}^{*}}\right) \in \mathbb{K}$ and, by omitting the arrow part of the object of $\mathbb{K}$, we put $p^{F}(g)=F\left(s G_{3} \otimes s G_{2}^{*} \otimes s G_{1}^{*}\right)$. Then, for any commutative diagram in $\mathbb{G}$

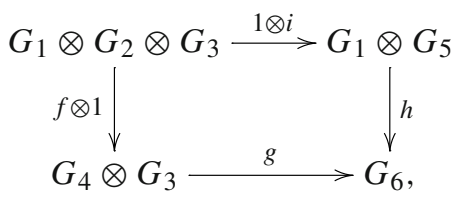

the morphism

$$
\begin{gathered}
F\left(s G_{4} \otimes s G_{2}^{*} \otimes s G_{1}^{*}\right) \otimes F\left(s G_{6} \otimes s G_{3}^{*} \otimes s G_{4}^{*}\right) \\
\qquad t_{f, g, h, i}^{F} \\
{ }_{G_{1} F\left(s G_{5} \otimes s G_{3}^{*} \otimes s G_{2}^{*}\right) \otimes F\left(s G_{6} \otimes s G_{5}^{*} \otimes s G_{1}^{*}\right)}
\end{gathered}
$$

is uniquely determined by the following commutative diagram 


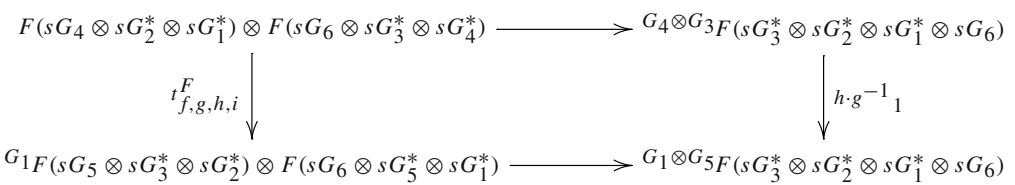

where the horizontal morphisms are suitably obtained applying $F_{2}$ and $F_{\varphi}$.

On the other hand, $\chi$ is determined, for any morphism $f: X \otimes Y \rightarrow Z$ in $\mathbb{E}$, by the morphism in $\mathbb{A}$

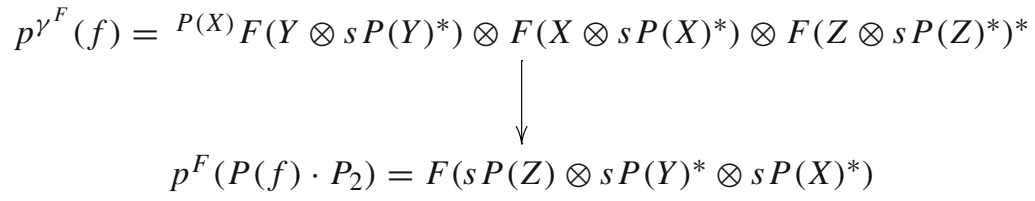

defined by means of $F_{\varphi}^{X, Y \otimes s P(Y)^{*}}, F_{2}, f^{*}: Z^{*} \longrightarrow Y^{*} \otimes X^{*}, F\left(X \otimes Y \otimes Z^{*}\right) \rightarrow I$, $F_{\varphi}^{X \otimes Y \otimes s P(Z)^{*}, s P(Z) \otimes s P(Y)^{*} \otimes s P(X)^{*}}$ and canonical morphisms.

On arrows $\tau=\left(\tau_{K}\right)_{K \in \mathbb{K}}: F \rightarrow F^{\prime}, \Phi(\tau):\left(\gamma^{F}, u_{\gamma^{F}}\right) \longrightarrow\left(\gamma^{F^{\prime}}, u_{\gamma^{\prime}}\right)$ is the arrow in $\mathbb{K e r}\left(\mathcal{T}_{P}\right)$ determined by $v \in \mathcal{C}^{1}(\mathbb{G}, \mathbb{A})$ given, for any $G \in \mathbb{G}$, by $v_{G}=I$ and the arrow $\rho: \gamma^{F} \longrightarrow P_{\mathcal{C}}(v) \otimes \gamma^{F^{\prime}}$ in $\mathcal{C}^{1}(\mathbb{E}, \mathbb{A})$ given, for any $X \in \mathbb{E}$, by the arrow in $\mathbb{A} \tau_{X \otimes s P(X)^{*}}: F\left(X \otimes s P(X)^{*}\right) \rightarrow F^{\prime}\left(X \otimes s P(X)^{*}\right)$.

To see that $\Phi$ is a monoidal functor is straightforward. Let us prove now that $\Phi$ is an equivalence.

To check that it is essentially surjective, suppose $\left(\gamma, u_{\gamma}\right)$ an object of $\mathbb{K} \operatorname{er}\left(\mathcal{T}_{P}\right)$. Then there is $(p, t) \in \mathcal{Z}^{2}(\mathbb{G}, \mathbb{A})$ and, for any $f: X \otimes Y \rightarrow Z$ in $\mathbb{G}$, a morphism in $\mathbb{A}, \theta_{f}: p^{\gamma}(f) \rightarrow p(P(f))$. Let us consider $F: \operatorname{Obj}(\mathbb{K}) \rightarrow \operatorname{Obj}(\mathbb{A})$ given, for any $\left(K, u_{K}\right) \in \mathbb{K}$, by $F\left(K, u_{K}\right)=\gamma_{K} \otimes p\left(u_{K} \cdot r\right)^{*}$. Next we see that there exist $F_{2}$ and $F_{\varphi}$ such that $F=\left(F, F_{2}, F_{\varphi}\right) \in \underline{\text { nf } \mathcal{H o m}}(\mathbb{K}, \mathbb{A})$.

For any morphism in $\mathbb{K},\left(K, u_{K}\right) \otimes\left(K^{\prime}, u_{K^{\prime}}\right) \stackrel{h}{\longrightarrow}\left(K^{\prime \prime}, u_{K^{\prime \prime}}\right)$ the existence of $F_{2}^{h}: F\left(K, u_{K}\right) \otimes F\left(K^{\prime}, u_{K^{\prime}}\right) \longrightarrow F\left(K^{\prime \prime}, u_{K^{\prime \prime}}\right)$ means the existence of a morphism $\gamma_{K} \otimes p\left(u_{K} \cdot r\right)^{*} \otimes \gamma_{K^{\prime}} \otimes p\left(u_{K^{\prime}} \cdot r\right)^{*} \longrightarrow \gamma_{K^{\prime \prime}} \otimes p\left(u_{K^{\prime \prime}} \cdot r\right)^{*}$. Now, thanks to $u_{\gamma}$ and $\theta_{h}$, there exists a morphism in $\mathbb{A}, \gamma_{K^{\prime}} \otimes \gamma_{K} \otimes \gamma_{K^{\prime \prime}}^{*} \longrightarrow p\left(P(h) \cdot P_{2}\right)$, and this one together with the morphism $t_{P(h) \cdot P_{2}, u_{K^{\prime \prime}} \cdot r, u_{K} \cdot r, u_{K^{\prime}} \cdot r}$, determined by the commutative square

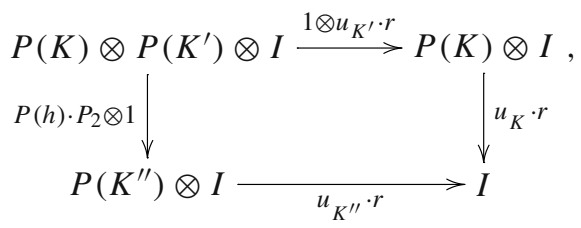

give the required morphism $F_{2}^{h}$. 
For any objects $X \in \mathbb{E}$ and $\left(K, u_{K}\right) \in \mathbb{K}$, the existence of $F_{\varphi}^{X,\left(K, u_{K}\right)}$ satisfying conditions (nf1), (nf2) and (nf3) means the existence of a morphism

$$
\Upsilon: \gamma_{X \otimes K \otimes X^{*}} \otimes p\left(u_{X \otimes K \otimes X^{*}} \cdot r\right)^{*} \longrightarrow{ }^{P(X)}\left(\gamma_{K} \otimes p\left(u_{K} \cdot r\right)^{*}\right)
$$

and this is obtained as follows. Considering the morphisms

$$
a_{X, K, X^{*}}^{-1}: X \otimes\left(K \otimes X^{*}\right) \rightarrow(X \otimes K) \otimes K^{*}, \quad 1_{K \otimes X^{*}} \quad \text { and } \quad \eta_{X}^{-1}: X \otimes X^{*} \rightarrow I
$$

and the following ones associated with them

$$
\begin{aligned}
& \theta_{a_{X, K, X^{*}}^{-1}}: P(X) \gamma_{K \otimes X^{*}} \otimes \gamma_{X} \otimes \gamma_{(X \otimes K) \otimes X^{*}}^{*} \longrightarrow p\left(P\left(a_{X, K, X^{*}}^{-1}\right) \cdot P_{2}\right), \\
& \theta_{1_{K \otimes X^{*}}}: \gamma_{X^{*}} \otimes \gamma_{K} \otimes \gamma_{K \otimes X^{*}}^{*} \longrightarrow p\left(P_{2}\right)
\end{aligned}
$$

and

$$
\theta_{\eta_{X}^{-1}}: P(X) \gamma_{X^{*}} \otimes \gamma_{X} \longrightarrow p\left(P\left(\eta_{X}^{-1}\right) \cdot P_{2}\right)
$$

we can deduce the existence of the following morphism

$$
\theta: \gamma_{(X \otimes K) \otimes X^{*}} \rightarrow^{P(X)} \gamma_{K} \otimes p\left(P\left(\eta_{X}^{-1}\right) \cdot P_{2}\right) \otimes{ }^{P(X)} p\left(P_{2}\right)^{*} \otimes p\left(P\left(a_{X, K, X^{*}}^{-1}\right) \cdot P_{2}\right)^{*}
$$

Now, finally, this morphism $\theta$ induces $\Upsilon$ thanks to the morphism

$$
\begin{aligned}
& { }^{P(X)} p\left(u_{K} \cdot r\right) \otimes p\left(P\left(\eta_{X}^{-1}\right) \cdot P_{2}\right) \otimes P(X) p\left(P_{2}\right)^{*} \otimes p\left(P\left(a_{X, K, X^{*}}^{-1}\right) \cdot P_{2}\right)^{*} \otimes p\left(u_{X \otimes K} \otimes X^{*} \cdot r\right)^{*} \\
& \downarrow \zeta \\
& \text { I }
\end{aligned}
$$

whose existence is easily deduced from the following morphisms:

$$
\begin{gathered}
t_{r \cdot\left(1 \otimes u_{K}\right), P\left(\eta_{X}^{-1}\right) \cdot P_{2}, \xi, P_{2}}: p\left(r \cdot\left(1 \otimes u_{K}\right) \otimes p\left(P\left(\eta_{X}^{-1}\right) \cdot P_{2}\right) \longrightarrow{ }^{P(X)} p\left(P_{2}\right) \otimes p(\xi),\right. \\
t_{P\left(a^{-1}\right) \cdot P_{2}, u_{X \otimes K \otimes X^{*} \cdot r, \xi, r}}: p\left(P\left(a^{-1}\right) \cdot P_{2}\right) \otimes p\left(u_{X \otimes K \otimes X^{*}} \cdot r\right) \longrightarrow p(\xi) \otimes I
\end{gathered}
$$

and

$$
t_{r \cdot\left(1 \otimes u_{K}\right), r, r, u_{K} \cdot r}: p\left(r \cdot\left(1 \otimes u_{K}\right)\right) \otimes I \otimes \longrightarrow I \otimes{ }^{P(X)} p\left(u_{K} \cdot r\right)
$$

respectively associated to the following commutative diagrams: 

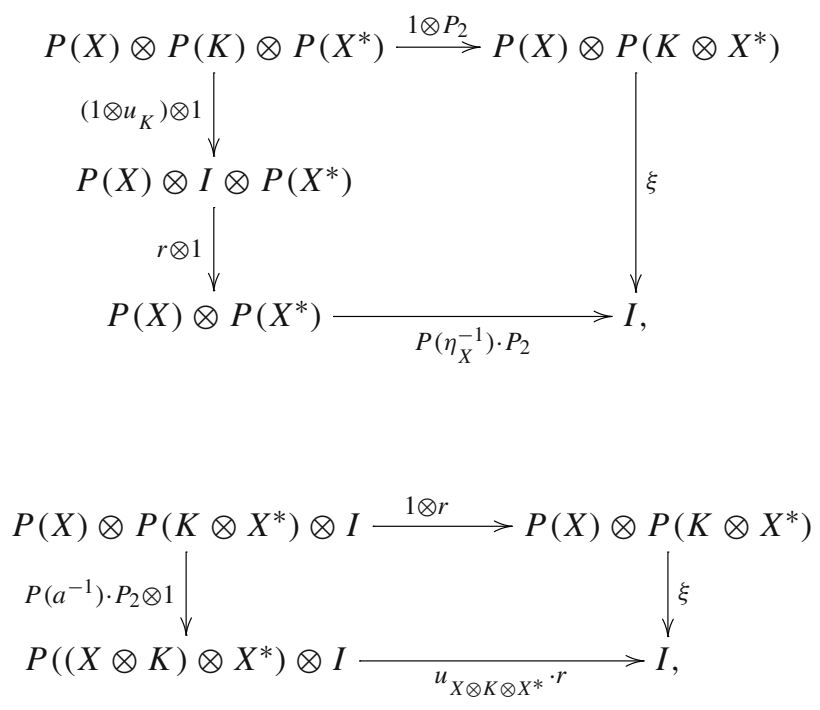

and

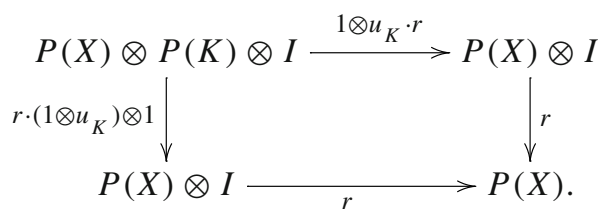

Moreover, we have that $\Phi(F)=\left(\gamma^{F}, u_{\gamma_{F}}\right)$ is isomorphic to $(\gamma, u)$ in $\mathcal{C}^{1}(P, \mathbb{A})$, the existence of this isomorphism being given by an object $v \in \mathcal{C}^{1}(\mathbb{G}, \mathbb{A})$ and, for any object $X \in \mathbb{E}$, by a morphism $\gamma_{X} \longrightarrow v_{P(X)} \otimes \gamma_{X}^{F}$ in $\mathbb{A}$, both described below. For any $X \in \mathbb{E}, \gamma_{X}^{F}=F\left(X \otimes s P(X)^{*}\right)=\gamma_{X \otimes s P(X)^{*}} \otimes p\left(u_{X \otimes s P(X)^{*}} \cdot r\right)^{*}$ and therefore we look for $v \in \mathcal{C}^{1}(\mathbb{G}, \mathbb{A})$ and, for any $X \in \mathbb{E}$, for a morphism

$$
\alpha: \gamma_{X} \longrightarrow v_{P(X)} \otimes \gamma_{X \otimes s P(X)^{*}} \otimes p\left(u_{X \otimes s P(X)^{*}} \cdot r\right)^{*}
$$

Now, on the one hand, consider the morphism

$$
\theta_{X \otimes s P(X)^{*}}:{ }^{P(X)} \gamma_{s P(X)^{*}} \otimes \gamma_{X} \otimes \gamma_{X \otimes s P(X)^{*}}^{*} \longrightarrow p\left(P_{2}\right)
$$

and the following one induced by it

$$
\tilde{\theta}: \gamma_{X} \longrightarrow \gamma_{X \otimes s P(X)^{*}} \otimes p\left(P_{2}\right) \otimes P(X)_{\gamma_{s}(X)^{*}}^{*}
$$

On the other hand, associated with the commutative diagram 


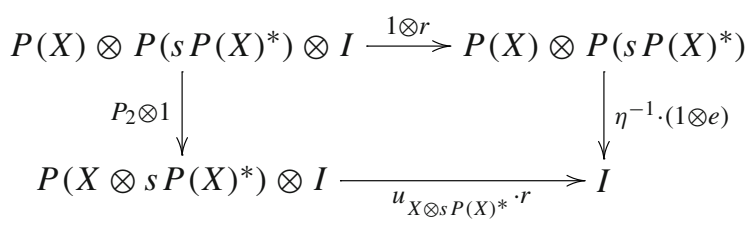

there is a morphism $p\left(P_{2}\right) \otimes p\left(u_{X \otimes s} P(X)^{*} \cdot r\right) \longrightarrow p\left(\eta^{-1} \cdot(1 \otimes e)\right)$ which induces, together with the above morphism $\widetilde{\theta}$, a new morphism

$$
\gamma_{X} \longrightarrow p\left(\eta^{-1} \cdot(1 \otimes e)\right) \otimes P(X) \gamma_{s P(X)^{*}}^{*} \otimes \gamma_{X \otimes s P(X)^{*}} \otimes p\left(u_{X \otimes s P(X)^{*}} \cdot r\right)^{*}
$$

This is then the desired morphism $\alpha$ if we consider $v \in \mathcal{C}^{1}(\mathbb{G}, \mathbb{A})$ given, for any $G \in \mathbb{G}$, by $v_{G}=p\left(\eta^{-1} \cdot(1 \otimes e)\right) \otimes{ }^{s G} \gamma_{s G^{*}}^{*}$ and this concludes the proof that $\Phi$ is essentially surjective.

It remains to prove that $\Phi$ is full and faithfull, that is, that for any $F, F^{\prime} \in$ $\underline{\mathrm{nf}} \mathcal{H} \mathrm{om}(\mathbb{K}, \mathbb{A})$ the $\operatorname{map}\left(F \stackrel{\tau}{\rightarrow} F^{\prime}\right) \mapsto\left(\Phi(F) \stackrel{\Phi(\tau)}{\longrightarrow} \Phi\left(F^{\prime}\right)\right.$ is bijective.

Recall that $\Phi(\tau):\left(\gamma^{F}, u_{\gamma^{F}}\right) \longrightarrow\left(\gamma^{F^{\prime}}, u_{\gamma^{\prime}}\right)$ is the class of the premorphism $(v, \rho)$ with $v \in \mathcal{C}^{1}(\mathbb{G}, \mathbb{A})$ given, for any $G \in \mathbb{G}$, by $v_{G}=I$, and $\rho: \gamma^{F} \longrightarrow$ $P_{\mathcal{C}}(v) \otimes \gamma^{F^{\prime}}$ in $\mathcal{C}^{1}(\mathbb{E}, \mathbb{A})$ is given, up to a canonical morphism, for any $X \in \mathbb{E}$, by the arrow in $\mathbb{A} \tau_{X \otimes s P(X)^{*}}: F\left(X \otimes s P(X)^{*}\right) \rightarrow F^{\prime}\left(X \otimes s P(X)^{*}\right)$.

Suppose $(w, h)$ any premorphism in $\mathbb{K} \operatorname{er}\left(\mathcal{T}_{P}\right)$ from $\Phi(F)$ to $\Phi\left(F^{\prime}\right)$. Thus, $w \in$ $\mathcal{C}^{1}(\mathbb{G}, \mathbb{A})$ and, for any $X \in \mathbb{E}$, there is a morphism in $\mathbb{A}, h_{X}: F\left(X \otimes s P(X)^{*}\right) \longrightarrow$ $w_{P(X)} \otimes F^{\prime}\left(X \otimes s P(X)^{*}\right)$. Moreover, the equality $u_{\gamma^{F^{\prime}}} \cdot \mathcal{T}_{P}(w, h)=u_{\gamma^{F}}$ must hold and this implies the existence of a morphism in $\mathcal{Z}^{2}(\mathbb{G}, \mathbb{A}),\left(p^{w}, t^{w}\right) \otimes\left(p^{F^{\prime}}, t^{F^{\prime}}\right) \longrightarrow$ $\left(p^{F}, t^{F}\right)$, that is, for any morphism $G_{1} \otimes G_{2} \rightarrow G_{3}$ in $\mathbb{G}$, the existence of a morphism

$$
{ }^{G_{1}} w_{G_{2}} \otimes w_{G_{1}} \otimes w_{G_{3}}^{*} \otimes F^{\prime}\left(s G_{3} \otimes s G_{2}^{*} \otimes s G_{1}^{*}\right) \longrightarrow F\left(s G_{3} \otimes s G_{2}^{*} \otimes s G_{1}^{*}\right) .
$$

In particular, considering the morphism $G \otimes P(s G)^{*} \stackrel{e}{\longrightarrow} P(s G) \otimes P(s G)^{*} \rightarrow I$, and taking into account that $w_{I}=I$ and $s I=I$, we find a morphism

$$
v: G_{w_{P(s G)^{*}}} \otimes w_{G} \otimes F^{\prime}\left(\left(s P(s G)^{*}\right)^{*} \otimes s G^{*}\right) \longrightarrow F\left(\left(s P(s G)^{*}\right)^{*} \otimes s G^{*}\right)
$$

Also, considering for any $G \in \mathbb{G}$ the morphism

$$
h_{s G^{*}}: F\left(s G^{*} \otimes\left(s P(s G)^{*}\right)^{*}\right) \longrightarrow w_{P(s G)^{*}} \otimes F^{\prime}\left(s G^{*} \otimes\left(s P(s G)^{*}\right)^{*}\right)
$$

and the one that it induces, ${ }^{P(s G)} h_{s G^{*}}$, we actually get a morphism

$$
\left.\left.F\left(\left(s P(s G)^{*}\right)^{*}\right) \otimes s G^{*}\right) \longrightarrow G_{P(s G)^{*}} \otimes F^{\prime}\left(\left(s P(s G)^{*}\right)^{*}\right) \otimes s G^{*}\right)
$$


This last morphism together with the above $v$ imply the existence, for any $G \in \mathbb{G}$, of a morphism $w_{G} \rightarrow I$. Then, for any $X \in \mathbb{E}, h_{X}$ induces a morphism

$$
\widetilde{h}_{X}: F\left(X \otimes s P(X)^{*}\right) \longrightarrow F^{\prime}\left(X \otimes s P(X)^{*}\right)
$$

and, in particular, for any object $\left(K, u_{K}\right) \in \mathbb{K}$, we obtain a morphism

$$
F(K) \otimes F\left(s P(K)^{*}\right) \stackrel{F_{2}}{\longrightarrow} F\left(K \otimes s P(K)^{*}\right) \stackrel{\widetilde{h_{K}}}{\longrightarrow} F^{\prime}\left(K \otimes s P(K)^{*}\right) \stackrel{F_{2}^{\prime-1}}{\longrightarrow} F^{\prime}(K) \otimes F^{\prime}\left(s P(K)^{*}\right)
$$

which determines the existence of $\tau_{K}: F(K) \longrightarrow F^{\prime}(K)$ with $\widetilde{h}_{K}=\tau_{K \otimes s P(K)^{*}}$ and such that, for any $X \in \mathbb{E}, \tau_{X \otimes s P(X)^{*}}=\widetilde{h}_{X}=h_{X}$. can. In this way we have that $\Phi(\tau)=\overline{(v, \rho)}=\overline{(w, h)}$ and so $\Phi$ is full.

Finally, if $\tau$ and $\tau^{\prime}$ are such that $\Phi(\tau)=\Phi\left(\tau^{\prime}\right)$ then, for any $X \in \mathbb{E}$, we have that $\tau_{X \otimes s P(X)^{*}}=\tau_{X \otimes s P(X)^{*}}^{\prime}$ and, in particular, for any $\left(K, u_{K}\right) \in \mathbb{K}$ we have that $\tau_{K} \otimes \tau_{s P(K)^{*}}=F_{2}^{\prime-1} \cdot \tau_{K \otimes s P(K)^{*}} \cdot F_{2}=F_{2}^{\prime-1} \cdot \tau_{K \otimes s P(K)^{*}}^{\prime} \cdot F_{2}=\tau_{K}^{\prime} \otimes \tau_{s P(K)^{*}}^{\prime}$ and therefore $\tau=\tau^{\prime}$ and so $\Phi$ is faithful.

Finally, Theorem 4.3 together with Proposition 4.2 give the following:

Corollary 1 (The Hochschild-Serre 2-exact sequences) Let $P: \mathbb{E} \rightarrow \mathbb{G}$ be an essentially surjective homomorphism of categorical groups with kernel $\mathbb{K}$ and let $\mathbb{A}$ be a symmetric $\mathbb{G}$-categorical group. There exist 2 -exact sequences of symmetric categorical groups

$$
\begin{aligned}
\mathcal{D}(\mathbb{G}, \mathbb{A}) \longrightarrow \mathcal{D}(\mathbb{E}, \mathbb{A}) \longrightarrow \underline{n f \mathcal{H o m}}(\mathbb{K}, \mathbb{A}) \longrightarrow \mathcal{H}^{2}(\mathbb{G}, \mathbb{A}) \longrightarrow \mathcal{H}^{2}(\mathbb{E}, \mathbb{A}) \\
\mathcal{H}^{1}(\mathbb{G}, \mathbb{A}) \longrightarrow \mathcal{H}^{1}(\mathbb{E}, \mathbb{A}) \longrightarrow \underline{n f \mathcal{H o m}}(\mathbb{K}, \mathbb{A}) \longrightarrow \underline{\mathcal{H}}^{2}(\mathbb{G}, \mathbb{A}) \longrightarrow \underline{\mathcal{H}}^{2}(\mathbb{E}, \mathbb{A}) .
\end{aligned}
$$

Note that, when $\mathbb{G}$ is the discrete categorical group associated with a group $G$ and $\mathbb{A}$ is the discrete symmetric categorical group associated with a $G$-module, these sequences reduce, by taking $\pi_{0}$, to the classic 5-term group exact sequences by Hochschild-Serre [14].

Acknowledgements I am indebted to E. M. Vitale helpful conversations for the development of this paper. Also, I am indebted to the referee for a number of valuable comments.

\section{References}

1. Baez, J.C., Lauda, D.A.: Higher dimensional algebra v: 2-groups. Theory Appl. Categ. 12, 423-491 (2004)

2. Bénabou, J.: Some remarks on 2-categorical algebra. Bulletin de la Société Mathématique de Belgique 41, 127-194 (1989)

3. Breen, L.: Théorie de Schreier supérieure. Annales Scientifiques de 1'Ecole Normale Supérieure 25, 465-514 (1992) 
4. Bullejos, M., Cegarra, A.M.: Categories through geometric nerves. Can. Math. Bull. 47, 321-331 (2004)

5. Carrasco, P., Cegarra, A.M.: (Braided) tensor structures on homotopy groupoids and nerves of (braided) categorical groups. Commun. Algebra 24(13), 3995-4058 (1996)

6. Carrasco, P., Cegarra, A.M.: Schreier theory for central extension of categorical groups. Commun. Algebra 24(13), 4059-4112 (1996)

7. Carrasco, P., Cegarra, A.M., Garzon, A.R.: The classifying space of a categorical crossed module. Math. Nachr. 283, 544-567 (2010)

8. Carrasco, P., Cegarra, A.M., Garzon, A.R.: Nerves and the classifying spaces for bicategories. Algebraic Geom. Topol. 10, 219-274 (2010)

9. Carrasco, P., Garzon, A.R., Miranda, J.G.: Schreier theory for singular extensions of categorical groups. Commun. Algebra 28, 2585-2613 (2000)

10. Carrasco, P., Garzon, A.R., Vitale, E.M.: On categorical crossed modules. Theory Appl. Categ. 16, 585-618 (2006)

11. del Rio, A., Martínez-Moreno, J., Vitale, E.M.: Chain complexes of symmetric categorical groups. J. Pure Appl. Algebra 196, 279-312 (2005)

12. Garzon, A.R., Inassaridze, H., del Rio, A.: Derivations of categorical groups. Theory Appl. Categ. 13, 86-105 (2004)

13. Garzon, A.R., Vitale, E.M.: On the second cohomology categorical group and a Hochschild-Serre 2-exact sequence. Theory Appl. Categ. 30(27), 933-984 (2015)

14. Hochschild, G., Serre, J.-P.: Cohomology of group extensions. Trans. Am. Math. Soc. 74, 110-134 (1953)

15. Johnson, N., Osorno, A.: Modeling stable one-types. Theory Appl. Categ. 26, 520-537 (2012)

16. Joyal, A., Street, R.: Braided tensor categories. Adv. Math. (1) 82, 20-78 (1991)

17. Kasangian, S., Metere, G., Vitale, E.M.: Split extensions, semidirect product, and holomorph of categorical groups. Homol. Homot. Appl. 8, 145-167 (2006)

18. Kasangian, S., Vitale, E.M.: Factorization systems for symmetric categorical groups. Theory Appl. Categ. 7, 47-70 (2000)

19. Mac Lane, S.: Homology. Die Grundlehren der Mathematischen Wissenschaften, vol. 114. Springer, Berlin (1963)

20. Rousseau, A.: Bicatégories monoidales et extensions de gr-catégories. Homol. Homot. Appl. 5, 437547 (2003)

21. Sinh, H.X.: Gr-catégories. Ph.D. Thesis, Université Paris VII (1975)

22. Takeuchi, M., Ulbrich, K.H.: Complexes of categories with abelian group structure. J. Pure Appl. Algebra 27, 61-73 (1983)

23. Ulbrich, K.H.: Group cohomology for Picard categories. J. Algebra 91, 464-498 (1984)

24. Vitale, E.M.: A Picard-Brauer exact sequence of categorical groups. J. Pure Appl. Algebra 175, 383408 (2002) 